\title{
Taxonomic revision of the South American subgenus Canthon (Goniocanthon) Pereira \& Martínez, 1956 (Coleoptera: Scarabaeidae: Scarabaeinae: Deltochilini)
}

\author{
Luis Gabriel de O.A. NUNES ${ }^{1, *}$, Rafael V. NUNES ${ }^{2} \&$ Fernando Z. VAZ-DE-MELLO $^{3}$ \\ ${ }^{1,2}$ Universidade Federal de Mato Grosso, Instituto de Biociências, Programa de Pós Graduação em \\ Ecologia e Conservação da Biodiversidade, Av. Fernando Correa da Costa, 2367, \\ Boa Esperança, Cuiabá-MT, 78060-900, Brazil. \\ ${ }^{3}$ Universidade Federal de Mato Grosso, Instituto de Biociências, Departamento de Biologia e \\ Zoologia, Av. Fernando Correa da Costa, 2367, Boa Esperança, Cuiabá-MT, 78060-900, Brazil.
}

*Corresponding author: luis.gabriel.bio@gmail.com

2Email: rafavnunes@gmail.com

${ }^{3}$ Email: vazdemello@gmail.com

\footnotetext{
${ }^{1}$ urn:1sid:zoobank.org:author:BBCA47BF-00F1-4967-8153-DC91FAE5A0E5

${ }^{2}$ urn:1sid:zoobank.org:author:9ED1789C-F1B2-499F-87B2-87B70FA9A2F3

${ }^{3}$ urn:1sid:zoobank.org:author:2FF2B7D6-1A6B-43C1-9966-A1A949FB2B05
}

\begin{abstract}
In this article, the subgenus Canthon (Goniocanthon) Pereira \& Martínez, 1956 is diagnosed within the tribe Deltochilini Lacordaire, 1856 and redefined with three species: 1) C. (Goniocanthon) bicolor Castelnau, 1840, from the Guyanas and northern South America, included for the first time in this subgenus; 2) C. (G.) smaragdulus (Fabricius, 1781), including two subspecies, C. (G.) smaragdulus smaragdulus, senior synonym of Canthon speculifer Castelnau, 1840 (neotype here designated), from the southern portion of the Atlantic Forest and $C$. (G.) smaragdulus subviridis Schmidt stat. rev. (lectotype here designated) from the northern portion of the Atlantic Forest; 3) C. (G.) fulgidus Redtenbacher, 1868, which includes three subspecies, C. (G.) fulgidus fulgidus from the southern Amazon (lectotype here designated), C. (G.) fulgidus martinezi subsp. nov., from the central and southern Amazon and C. (G.) fulgidus pereirai subsp. nov., from the western Amazon.
\end{abstract}

Keywords. Dung beetles, Amazon rainforest, Atlantic Forest, new subspecies, rollers.

Nunes L.G. de O.A., Nunes R.V. \& Vaz-de-Mello F.Z. 2018. Taxonomic revision of the South American subgenus Canthon (Goniocanthon) Pereira \& Martínez, 1956 (Coleoptera: Scarabaeidae: Scarabaeinae: Deltochilini). European Journal of Taxonomy 437: 1-31. https://doi.org/10.5852/ejt.2018.437

\section{Introduction}

Until recently, the tribe Deltochilini Lacordaire, 1856, spread all around the world, included nearly 120 described genera (Scholtz 2009; cited as Canthonini van Lansberge, 1875). In 2016, a new definition for the tribe was proposed, which restricted the group to 22 genera from the Neotropical and Nearctic 
regions (Tarasov \& Dimitrov 2016). Among those genera, Canthon Hoffmannsegg, 1817 is one of the most diverse, including 174 described species (Halffter \& Martínez 1977). Most of these species are organized in the following subgenera: Canthon s. str. (63 spp), Glaphyrocanthon Martínez, 1948 (42 spp.), Peltecanthon Pereira, 1953 (3 spp.), Pseudepilissus Martínez, 1954 (7 spp.), Goniocanthon Pereira \& Martínez, 1956 (3 spp.), Nesocanthon Pereira \& Martínez, 1956 (3 spp.), Boreocanthon Halffter, 1958 (15 spp.), Francmonrosia Pereira \& Martínez, 1959 (10 spp.) and Trichocanthon Pereira \& Martinez, 1959 (monospecific). Twenty-six other species are still considered as incertae sedis in the genus Canthon (see Halffter \& Martínez 1977; Vaz-de-Mello et al. 2011).

Goniocanthon was originally described as a genus, including Scarabaeus smaragdulus Fabricius, 1781, which was designated as type species, and Canthon fulgidus Redtenbacher, 1868. Subsequently, Halffter \& Martínez (1977) downgraded Goniocanthon to subgeneric level under Canthon. Since then, Canthon (Goniocanthon) has been considered as belonging to the tribes of Canthonini or Deltochilini because of the following character combination: dorsal surface (pronotum and elytra) smooth with colourful metallic sheen and lacking tubercles, metasternum anterior lobe and pygidium convex (Pereira \& Martínez 1956; Halffter \& Martínez 1977; Vaz-de-Mello et al. 2011). Four names are currently recognized: C. smaragdulus (Fabricius, 1781), C. speculifer Castelnau, 1840, C. speculifer subviridis Schmidt, 1922 stat. rev. and C. fulgidus Redtenbacher, 1868.

Both C. smaragdulus and C. fulgidus have a noticeable dorsal colour variation related to their geographic distribution. Individuals of Canthon smaragdulus from the northeastern Atlantic Forest in Brazil have a green colour, in contrast to blue-coloured individuals from the southern Atlantic Forest. Canthon fulgidus also exhibits chromatic variation in the Amazon: a green phenotype occurs in Colombia, Ecuador, Peru and Brazil (only in Amazonas State) and the species is commonly misidentified both in collections and in publications as C. smaragdulus (e.g., Pessôa \& Lâne 1941; Blackwelder 1944; Vulcano \& Pereira 1964; Medina et al. 2001; Ratcliffe et al. 2015).

The aim of this work is to reassess the taxonomic problems related to Goniocanthon through a taxonomic revision of the subgenus. This revision provides a better and clearer delimitation of the subgenus and its species through descriptions, diagnoses, identification key, distribution maps and illustrations.

\section{Material and methods}

Specimens from the following institutions were examined (curator in parentheses):

NHMUK $=$ The Natural History Museum, London, United Kingdom (Max Barclay). The code "BMNH" is used on the specimen labels.

CMN = Canadian Museum of Nature, Ottawa, Canada (François Génier). The code "CMNC" is used on the specimen labels.

UFMI = Setor de Entomologia da Coleção Zoológica da Universidade Federal de Mato Grosso, Cuiabá, Brazil (Fernando Z. Vaz-de-Mello). The code "CEMT" is used on the specimen labels.

FAUN = Colección Entomológica de La Universidad de Nariño, Pasto, Colombia (Mauricio Rodríguez). The code "CEUN" is used on the specimen labels.

HMUG = The Hunterian Museum, Glasgow, United Kingdom (examined in Oxford through a loan to Darren Mann)

RBINS = Institut royal des Sciences naturelles de Belgique, Brussels, Belgium (Alain Drumont). The code "ISNB" is used on the specimen labels.

MNHN = Muséum national d'Histoire naturelle, Paris, France (Olivier Montreuil and Antoine Mantilleri)

MZSP = Museu de Zoologia, Universidade de São Paulo, São Paulo, Brazil (Sonia Casari and Carlos Campaner)

NMW = Naturhistorisches Museum of Wien, Vienna, Austria (Harald Schillhammer)

NMPC = National Museum (National History), Prague, Czech Republic (Jiřŕ Hájek) 
NUNES L.G. DE O.A. et al., Revision of the South American subgenus Canthon (Goniocanthon)

NHRS = Naturhistoriska Riksmuseet, Stockholm, Sweden(Johannes Bergsten and Mattias Forshage)

OUM = Oxford Museum of Natural History, Oxford, United Kingdom (Darren J. Mann). The code "OUMNH" is used on the specimen labels.

ZMHU = Museum für Naturkunde der Humboldt-Universität, Berlin, Germany, (Joachim Willers and Johannes Frisch). The code "ZMHB" is used on the specimen labels.

Lists of examined material were separated into type material and non-type material. For type material, labels are transcribed ipsis litteris and are separated by "/", with species names in italics. After the information from each type's labels, label colour or main characteristics are briefly explained in parentheses. In non-type material lists, information retrieved from labels was organized as follows: country names, or geographical extent of countries located in other continents, in capital letters (e.g., BRAZIL); department or state names are separated by an n-dash; the number of males ( $\hat{\delta}$ or $\delta^{\lambda}$ ) and females ( $q$ or $q Q$ ); municipality and/or locality (when available); coordinates, collection techniques, environment/ecological information, date (e.g., 3 Mar. 1986 means March $3^{\text {rd }}$, 1986) and collector (leg.). When available, collection techniques or other details were transcribed in the original language found on the label. The collection where the material is deposited is given in brackets (e.g., (CEMT) means deposited in CEMT collection).

Abbreviations:

$\mathrm{A} \quad=$ axial sclerite

$\mathrm{BL}=$ body length

FLP $=$ fronto-lateral peripheric sclerite

$\mathrm{MP}=$ medial peripheric sclerite

$\mathrm{PW}=$ pronotum width

$\mathrm{SA}=$ subaxial sclerite

$\mathrm{SRP}=$ superior peripheric sclerite

$\mathrm{T}=$ temones

Extraction and preservation of internal sac and its sclerites were done according to the procedures described in the Scarabaeinae literature (Zunino 1978; Tarasov \& Solodovnikov 2011). External morphology terminology was based on recently published taxonomic and phylogenetic works dealing with the tribe Deltochilini and/or the genus Canthon (Vaz-de-Mello et al. 2011; Tarasov \& Génier 2015).

Behavioural and ecological data described for C. fulgidus were obtained in Rondônia state localities between 2011 and 2015.

\section{Results}

Classe Hexapoda Blainville, 1816

Order Coleoptera Linnaeus, 1758

Family Scarabaeidae Latreille, 1802

Subfamily Scarabaeinae Latreille, 1802

Tribe Deltochilini Lacordaire, 1856

Canthon (Goniocanthon) Pereira \& Martínez, 1956

Goniocanthon Pereira \& Martínez, 1956: 109.

Canton (Goniocanthon) Halffter \& Martinez, 1977: 74.

Goniocanthon - Martinez 1959: 49 (catalogue). - Halffter 1961: 231 (monograph). — Vulcano \& Pereira 1964: 592 (catalogue). 
Canthon (Goniocanthon) - Vaz-de-Mello 2000: 191 (species list). — Medina et al. 2001: 136 (species list). — Vaz-de-Mello et al. 2011: 4, 12, 19, 26, 34, 42 (key).

\section{Type-species}

Canthon smaragdulus (Fabricius, 1781), by original designation.

\section{Diagnosis}

Among Scarabaeinae Latreille, 1802, the tribe Deltochilini is characterized by having wing venation Radio Posterior 1 (RP1) with small basal appendix; venation Radio Anterior 4 (RA4) significantly thinner than RP1, and RA4 fused basally with RP1 (Tarasov \& Dimitrov 2016). Within the tribe, the subgenus Goniocanthon is differentiated by the following combination of characters: two clypeal teeth; pronotal and elytral discs smooth, shiny and lacking lateral tubercles or carinae; hypomeron lacking transverse keel; metasternal anterior lobe convex or flat; pygidium convex; dorso-ventral surfaces of the body bearing metallic sheen; and ventral surface of metafemur not marginate anteriorly (Halffter \& Martínez 1977; Vaz-de-Mello et al. 2011).

\section{Key to species and subspecies of Canthon (Goniocanthon) Pereira \& Martínez, 1956}

1. Pygidium strongly convex. Metasternal anterior lobe convex or flat (Fig. 1). Dorsal surface glabrous with blue, green, golden, or red metallic sheen (Figs 2-4). Ventral surface of metafemur not marginate anteriorly .................2 Canthon (Goniocanthon) Pereira \& Martínez, 1956

- Pygidium flat or slightly convex. Metasternal anterior lobe, if protuberant, angled or truncated, not convex. Dorsal surface pubescent or glabrous, with or without metallic sheen. Ventral surface of metafemur marginate or not anteriorly ...

......other subgenera and isolated species-groups of Canthon sensu Halffter \& Martínez (1977)

2. Both pygidium and metasternal anterior lobe strongly convex. Profemur with a tooth on its anterior lower border. Pronotum and elytra with similar colour. South America ..............3

- Only pygidium convex (Fig. 4C). Metasternal anterior lobe flat (Fig. 4B). Profemur without tooth on its anterior border (Fig. 4B). Pronotum metallic green and elytra bright black (Fig. 4A). Guyanas, Suriname, Venezuela ..............................C. (G.) bicolor Castelnau, 1840

3. Meso- and metafemora ventral surface completely smooth (Fig. 1A). Metasternal anterior lobe strongly convex in males (Fig. 1B). Dorsal surface with blue or green metallic sheen (Fig. 2). Brazil (from Ceará to Santa Catarina, westward till southern Mato Grosso do Sul), Argentina and Paraguay, along the Paraná River C. (G.) smaragdulus (Fabricius, 1781)

A Dorsal surface with blue metallic sheen (Fig. 2A). Brazilian coastal Atlantic Forest (from southern Rio de Janeiro to Santa Catarina, westward to southern Mato Grosso do Sul), Argentina and Paraguay along the Paraná River .............. (G.) smaragdulus smaragdulus (Fabricius, 1781)

A' Dorsal surface with green metallic sheen (Fig. 2B). Brazilian coastal Atlantic Forest (from Ceará to São Paulo) C. (G.) smaragdulus subviridis Schmidt, 1922 stat. rev.

- Meso- and metafemora ventral surface rugose on posterior half (Fig. 1D). Metasternal anterior lobe only slightly convex in males (Fig. 1E). Dorsal surface with green, golden, or red metallic sheen (Fig. 1E). Amazon forest in Bolivia, Brazil, Colombia, Ecuador and Peru

C. (G.) fulgidus Redtenbacher, 1868

B Dorsal surface with only red metallic sheen, without green or golden reflexes (Fig. 3C). Northeastern Bolivia. Brazil (Acre, northern Mato Grosso, southern and central Pará, Rondônia) C. (G.) fulgidus pereirai subsp. nov.

B' Dorsal surface with golden metallic sheen (Fig. 3A). Brazil (northern Mato Grosso) 

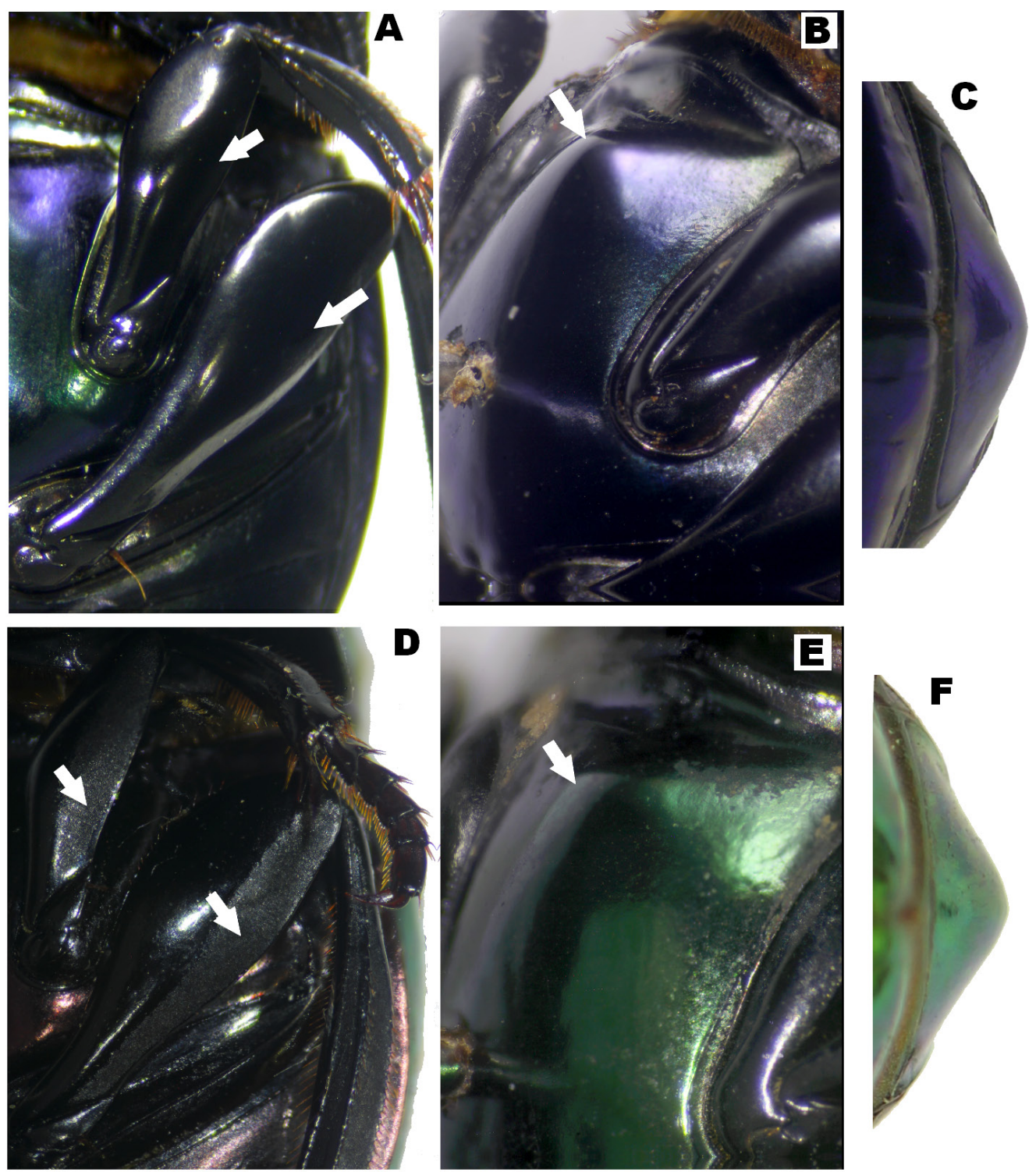

D
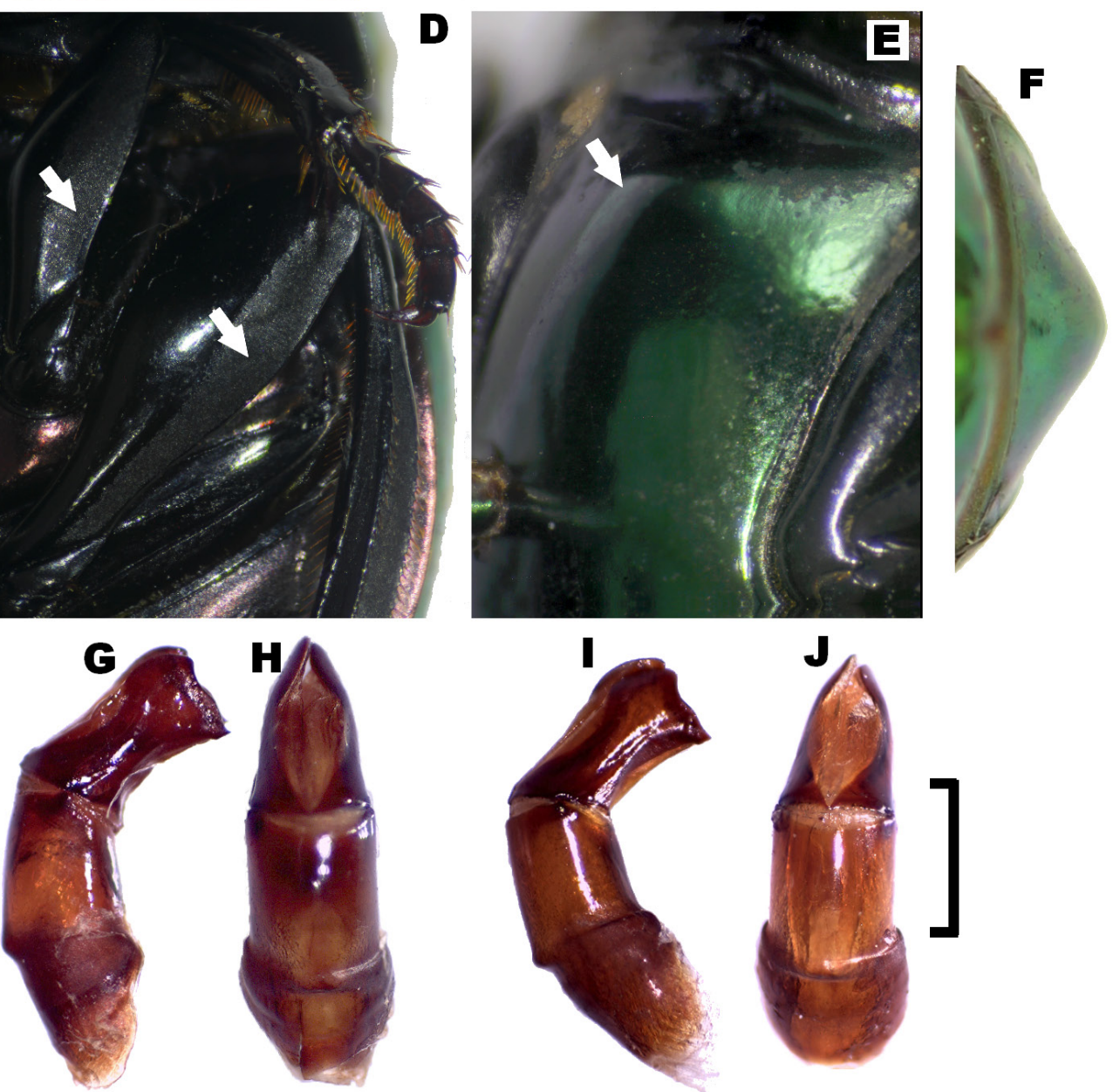

Fig. 1. A-C, G-H. Canthon (G.) smaragdulus smaragdulus (Fabricius, 1781). - D-F, I-J. Canthon (G.) fulgidus fulgidus Redtenbacher, 1868. A, D. Ventral surface of meso- and metafemora. B, E. Metasternal anterior lobe convex. C, F. Dorsal view showing convex pygidium. G, I. Paramera lateral view. H, J. Paramera dorsal view. Scale bar: $2.0 \mathrm{~mm}$. 
B" Dorsal surface with green metallic sheen, devoid of any orange, with sometimes slight golden reflection (Fig. 3B). Central Colombia, eastern Ecuador and Peru, and Brazil (western Amazonas) ............................................ (G.) fulgidus martinezi subsp. nov.

Canthon (Goniocanthon) smaragdulus (Fabricius, 1781)

Figs $1 \mathrm{~A}-\mathrm{C}, \mathrm{G}-\mathrm{H}, 2,5-6,8$

\section{Redescription}

\section{Males}

Colour. Dorsal and ventral surface green to blue, always with blue metallic sheen.

HEAD. Frontal surface smooth, lacking horns or tubercles. Clypeal teeth with rounded apex, with thin setae at the apex; teeth separated by a U-shaped emargination. Clypeo-genal junction slightly emarginated, rounded. Clypeo-genal suture present.

Pronotum. Disc simply convex, smooth, lacking microscultpure $(120 \times$ magnification). Anterior and posterior angles acute. Lateral borders effaced. Posterior border with lateral semicircular emarginations near to posterior angles.

HYPOMERON. Lacking transverse keel, surface black with chagrined microsculpture. Sparse setae present on anterior portion.

Metasternum. Anterior lobe distinctly convex (Fig. 1B).

ELYTRA. Striae weakly impressed, more distinct near elytral basis. Eighth stria carinate along its basal third. Interstriae flat.

LEGS. Protibiae three-toothed, dorsal surface with two tufts of setae present on each tibial tooth. Protibial spur truncate, wider than long and apically emarginated, forming two spines. External border of protibia serrated. Profemur ventral surface black, smooth, glabrous, with a distal tooth on its anterior border. Ventral surface of meso- and metatibiae smooth (Fig. 1A). Meso- and metatrochanter with a hair tuft near femur articulation.

ABDomen. Ventrites glabrous, lacking punctures and not narrowed medially.

Pygidium. Convex (Fig. 1C). Basal line delimiting pygidium from pro-pygidium.

Paramera. On lateral view (Fig. 1G). Flattened; apex truncated; upper angle rounded; lower angle acute, forming a spine or tooth. On dorsal view (Fig. 1H): membranous portion wider at central portion and narrower near apex and basis. Apex rounded.

INTERNAL SAC (Figs 5, 9). FLP involving A, forming a central duct with spiniform apex. SA lateral to FLP + A complex. MP distal to A, semicircular, widely emarginated; FLP + A complex involved by MP. SRP with lateral prolongation.

\section{Morphological variation}

BL: 9-14 mm, PW: 6-9 mm. Colouration of dorsal surface metallic sheen may vary from green to blue metallic colouration (Fig. 2A-B) (in both natural or artificial light). Female protibial spur as wide as long with a U-shaped emargination. Pygidium and metasternum flatter in females than in males. 
NUNES L.G. DE O.A. et al., Revision of the South American subgenus Canthon (Goniocanthon)

Canthon (Goniocanthon) smaragdulus smaragdulus (Fabricius, 1781)

Figs $1 \mathrm{~A}-\mathrm{C}, \mathrm{G}-\mathrm{H}, 2 \mathrm{~A}, 6,8$

Scarabaeus smaragdulus Fabricius, 1781: 34.

Canthon speculifer Castelnau, 1840: 68.

Scarabaeus smaragdulus - Fabricius 1787: 18 (catalogue). - Jablonsky \& Herbst 1789: 328 (catalogue). - Olivier 1789: 158 (catalogue). — Fabricius 1792: 70 (catalogue).

Ateuchus smaragdulus - Fabricius 1801: 58.

Coprobius smaragdulus - Brullé 1838: 294.

Canthon smaragdulus - Blanchard 1846: 161. - Harold 1868: 14, 75 (key). - Gemminger \& Harold 1869: 994 (catalogue). — Harold 1875: 59 (catalogue). — Gillet 1911: 33 (catalogue). — Schmidt 1922: 69 (key). - Boucomont 1928: 2 (catalogue). - Staig 1931: 44 (redescription; lectotype designation). — Balthasar 1939: 203 (key). — Pessôa \& Lâne 1941: 420 (species list). — Lange 1947: 307 (catalogue).

Canthon speculifer - Harold 1868: 13, 70 (key). — Gemminger \& Harold 1869: 994 (catalogue). Harold 1875: 58 (catalogue). - Heyne \& Taschenberg 1908: 61 (catalogue). — Boucomont 1928: 2 (catalogue). — Balthasar 1939: 203 (key). — Pessôa \& Lâne 1941: 420 (species list). — Blackwelder 1944: 201 (catalogue). — Lange 1947: 307 (catalogue). — Gacharná 1951: 221 (catalogue). — Guérin 1953: 257 (catalogue). - Pereira \& Martínez 1956: 111 (synonym of Goniocanthon smaragdulus).

Canthon smaragdulum - Blackwelder 1944: 201 (catalogue). — Louzada 1998: 125 (behaviour).

Goniocanthon smaragdulus - Pereira \& Martínez 1956: 109. — Vulcano \& Pereira 1964: 593 (catalogue). Canthon (Goniocanthon) smaragdulus - Halffter \& Martínez 1977: 75. — Vaz-de-Mello et al. 2011: 52-53, figs 35, 44 (key).

Canthon (Goniocanthon) smaragdulum - Vaz-de-Mello 2000: 191 (species list).

\section{Diagnosis}

Canthon (G.) smaragdulus smaragdulus is separated within the subgenus by the following combination of characters: ventral surface of meso- and metafemora smooth (Fig. 1A); dorsal surface of pronotum and elytral disc with similar blue metallic sheen colouration (Fig. 2A); metasternal anterior lobe strongly convex (Fig. 1B); profemur with a tooth on its anterior border. Paramera in lateral view (Fig. 1G): flattened; apex truncate; upper angle rounded; lower angle acute, forming a spine or tooth. Internal sac (Fig. 6): FLP involving A forming a central duct with spiniform apex. SA lateral to FLP + A complex present. MP distal to A, not divided, semicircular, widely emarginated; FLP + A complex involved by MP.

\section{Material examined}

Lectotype (of Scarabaeus smaragdulus Fabricius, 1781; designated by Staig 1931: 44) BRAZIL: $\widehat{0}$, type (round, white with red margin label)/141815 (white)/ compared with modern exemplars in Brit. Mus. R.A.S. March 1925 (white handwritten in red) / Hab. in Brasilia (white with black margins) / Sc. smaragdulus, Fabr. faog. $34 \mathrm{~N}^{\circ} 127$ (white with black margins) / Scarabaeus smaragdulus Fabr., Canthon smaragdulus Fabr. Type (white with red margins) / LECTOTYPE, Scarabaeus smaragdulus Fabricius, des. FZ Vaz-de-Mello 2014 (red with black margins) (HMUG 141815).

Neotype (for Canthon speculifer Castelnau, 1840; here designated)

BRAZIL: Õ, Muséum Paris, Rio Castelnau (white label) / 10, 44 (round white) / Canthon smaragdulus Fabr. (white) / NEOTYPE, Canthon speculifer Castelnau, des. FZ Vaz-de-Mello 2014 (red with black margins) (MNHN). 
Non-type material (125 specimens)

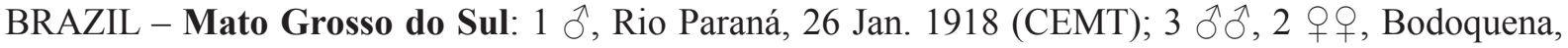

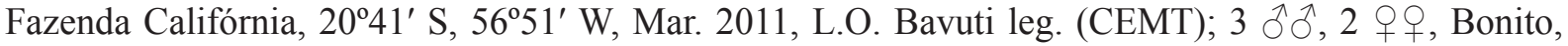

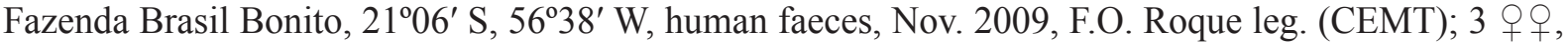
Bonito, Hotel Cabanas, 21 ${ }^{\circ} 10^{\prime} 15^{\prime \prime}$ S, 56 $26^{\prime} 22^{\prime \prime}$ W, human faeces, Dec. 2010, L.O. Bavuti leg. (CEMT);

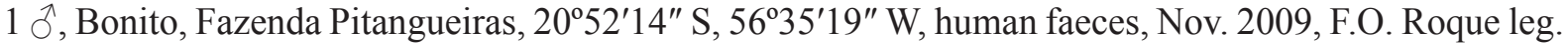

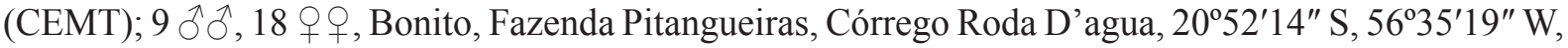

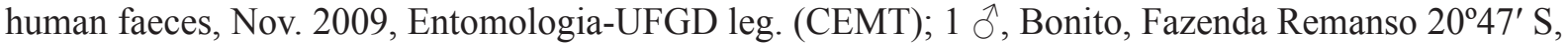

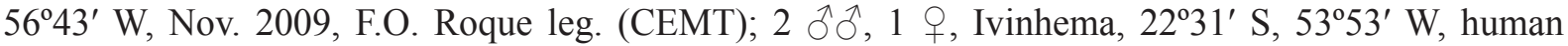

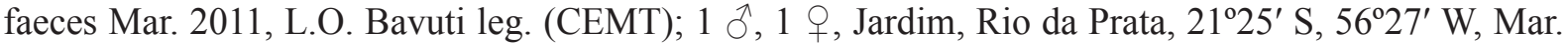
2011, L.O. Bavuti leg. (CEMT). - São Paulo: 1 đ̂, 1 , P.E. Serra do Mar, Núcleo Caraguatatuba, $23^{\circ} 35^{\prime} 27^{\prime \prime} \mathrm{S}, 45^{\circ} 25^{\prime} 19^{\prime \prime} \mathrm{W}$, human faeces, 17, 13 Jan. 2012, E. Bovy leg. (CEMT); 4 $\widehat{\jmath}, 1$ ㅇ, P.E. Serra do Mar, Núcleo Picinguaba, 2320'13" S, 4450'06" W, human faeces, 14 Jan. 2012, E. Bovy (CEMT);
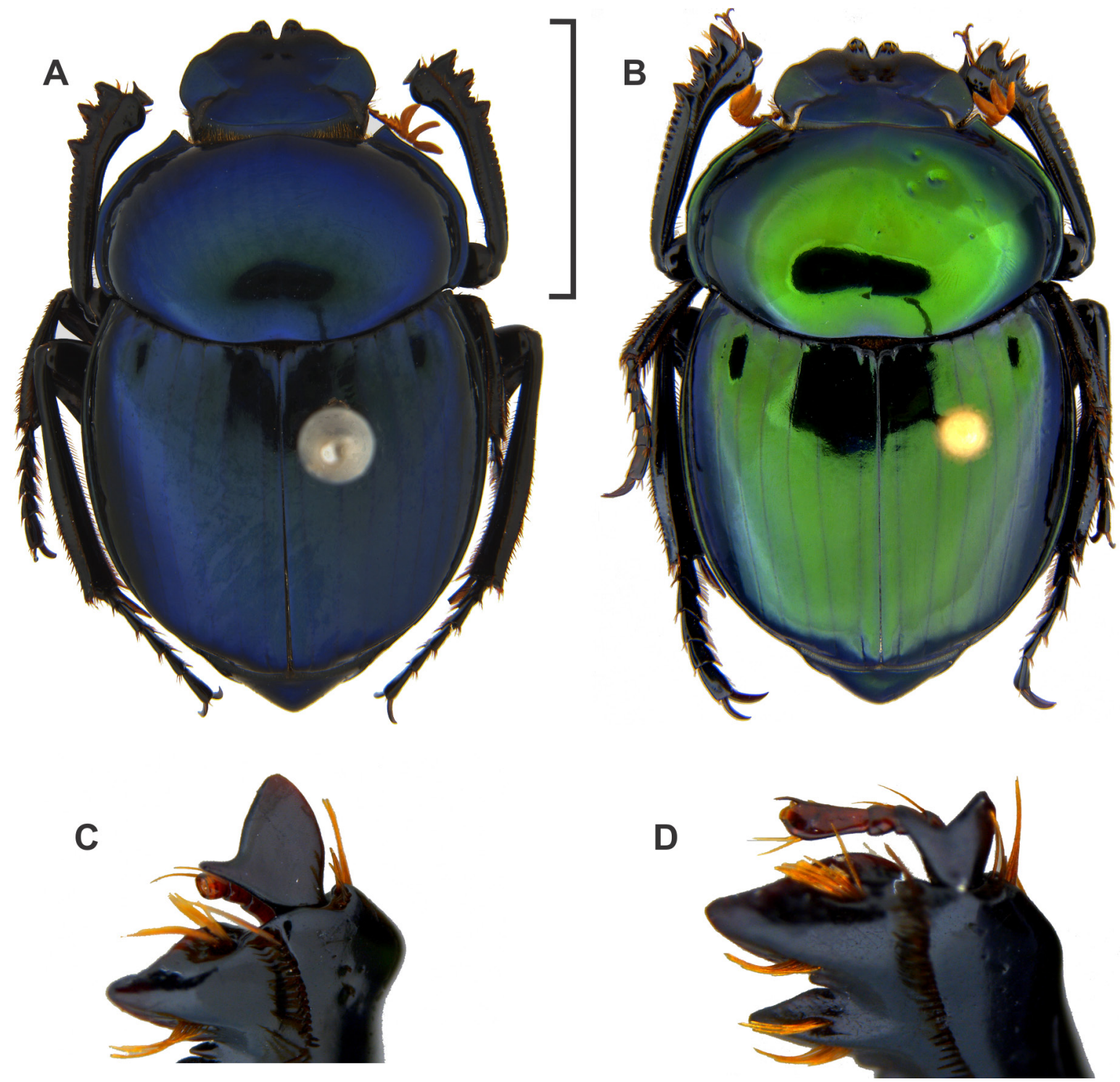

Fig. 2. A. Canthon (G.) smaragdulus smaragdulus (Fabricius, 1781). - B-D. C. (G.) smaragdulus subviridis Schmidt, 1922 stat. rev. A-B. Habitus. C. Male protibial spur. D. Female protibial spur. Scale bar: $\mathrm{A}-\mathrm{B}=5.0 \mathrm{~mm}$. 
5 우, same data as for preceding, but $23^{\circ} 20^{\prime} 11^{\prime \prime} \mathrm{S}, 44^{\circ} 50^{\prime} 03^{\prime \prime} \mathrm{W}$ (CEMT); 1 $\mathrm{O}^{\prime}, 1$, , same data as for preceding, but $23^{\circ} 20^{\prime} 05^{\prime \prime} \mathrm{S}, 44^{\circ} 49^{\prime} 59^{\prime \prime} \mathrm{W}$ (CEMT); 1 \%, same data as for preceding, but $23^{\circ} 20^{\prime} 07^{\prime \prime} \mathrm{S}$,

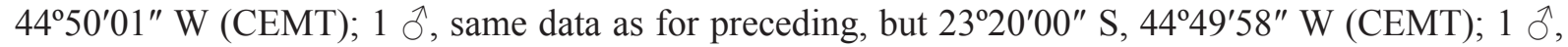

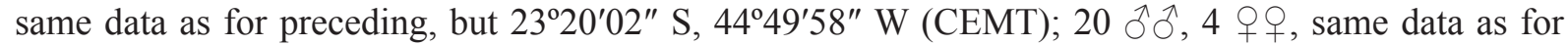

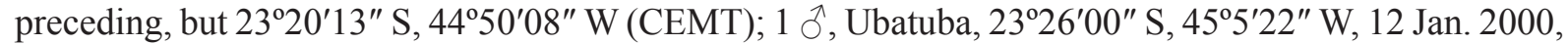
E. Pereira Coleção, G.P. Almeida leg. (CEMT); 1 đ, Campinas, Ribeirão Cachoeira, Mata, Jan. 1996, D.A. Gaspar leg. (CEMT); 2 đ̊̄, Campinas, Ribeirão Cachoeira, Mata, Fezes Alouatta, 5 Feb. 1998, Fabri and Setz leg. (CEMT); 1 o, 2 q $q$, Sete Barras, Parque Estadual Carlos Botelho, human faeces,

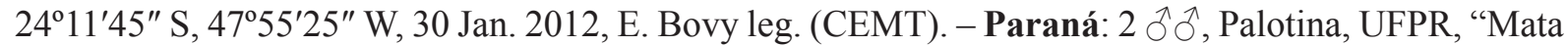
anexa ao campus", Pitfall 2, 28 Dec. 2011, R.J. Simioni, S.B. Silva and E. Caron leg. (CEMT); 4 ðð, 2 우, Morretes, Santuário Nhundiaquara, $25^{\circ} 25^{\prime} 32^{\prime \prime}$ S, 48 $53^{\prime} 21^{\prime \prime}$ W, 100 m a.s.1., human faeces, 15 Jan.

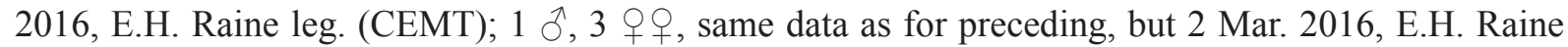

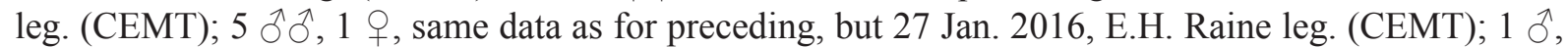
same data as for preceding, but $25^{\circ} 27^{\prime} 55^{\prime \prime} \mathrm{S}, 48^{\circ} 52^{\prime} 47^{\prime \prime} \mathrm{W}, 110 \mathrm{~m}$ a.s.l., human faeces, 3 Mar. 2016, E.H.

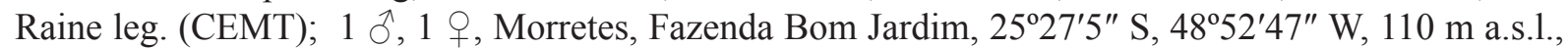
human faeces, 16 Jan. 2016, E.H. Raine leg. (CEMT); 1 ô, same data as for preceding, but pig faeces,

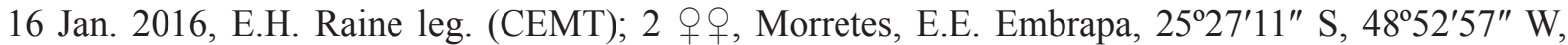
140 m a.s.l., human faeces, 17 Dec. 2015, E.H. Raine leg. (CEMT). - Rio: 1 ô, 1844, Castelnau leg.

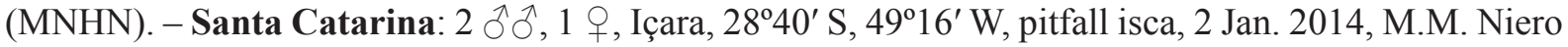
leg. (CEMT).

ARGENTINA - Misiones: 2 우, Puerto Iguazu, Jan. 1991 (CEMT); 1 O, Porto Libertad, APSA, P.P. Península, “bosque nativo adyacente a Pinar joven - 2007”, Feb. 2010, Coprotrampa, M.N. Peyras leg. (CEMT).

PARAGUAY - Guairá: 1 , General Eugenio Alejandrino Garay, 25-31 May 1992, U. Drechsel leg. (CEMT).

\section{Remarks and requirements for neotype designation according to Article 75 of The Code}

Canthon speculifer was described by Castelnau (1840: 68). From this description it is possible to confirm that Castelnau referred to a blue-to-green Goniocanthon, mainly because he cited the gibbose metasternum, unique among Canthon. Evenhuis (2012) stated that part of Castelnau's syntype collection was incorporated into the Smithsonian Institute in Washington, and destroyed in a fire on January $24^{\text {th }}$, 1865. Canthon speculifer was probably among these type specimens because successive searches in European and American museums did not yield the type series of $C$. speculifer. However, Castelnau had visited Brazil on a different occasion in the company of Eugêne d'Osery. They arrived in Rio Janeiro on June $17^{\text {th }}, 1843$ to acquire information about Brazilian geography and history, as well as specimens for the French Academy of Sciences (Papavero 1971). Among these is a historical specimen deposited at MNHN in 1844 and identified by Castelnau as C. speculifer. This specimen, which we now propose as neotype, is not part of the syntypic series because it was collected some time after the description of C. speculifer; however, it was Castelnau himself who identified and labelled it only shortly after his original description, thus he probably maintained a clear vision of $C$. speculifer. The comparison of the designated neotype with the lectotype of $C$. smaragdulus reveals that $C$. speculifer and $C$. smaragdulus are synonyms. This neotype designation fixes the name $C$. speculifer according to Castelnau's original concept and allows us to establish the synonymy with C. smaragdulus.

\section{Distribution}

Brazilian Atlantic Forest and Paraná river forest (states of Mato Grosso do Sul, Paraná, Santa Catarina, São Paulo and Rio de Janeiro). Also in the Argentinian province of Misiones and the Paraguayan department of Guairá (Fig. 8). 


\section{Taxonomic remarks}

The lectotype of Scarabaeus smaragdulus Fabricius, 1781 was designated by Staig (1931), who illustrated a specimen of the original series calling it "the type" (ICZN 1999: article 74.6).

Canthon (Goniocanthon) smaragdulus subviridis Schmidt, 1922 stat. rev. Figs 2B-D, 5, 8

Canthon speculifer var. subviridis Schmidt, 1922: 69.

Canthon speculifer var. subviridis - Blackwelder 1944: 201 (catalogue).

Canthon (Goniocanthon) smaragdulum v. subviride - Vaz-de-Mello 2000: 191 (species list).

\section{Diagnosis}

In the subgenus, $C$. (G.) smaragdulus subviridis stat. rev. is separated by the following combination of characters: ventral surface of meso- and metafemora smooth (Fig. 1A); dorsal surface of pronotum and elytral disc with similar metallic green colouration (Fig. 2B); metasternum anterior lobe strongly convex (Fig. 1B); profemur with a tooth on its anterior border. Paramera: on lateral view (Fig. 1G): flattened; apex truncated; upper angle rounded; lower angle acute, forming a spine or tooth. Internal sac (Fig. 5): FLP involving A forming a central duct with spiniform apex. SA lateral to FLP + A complex present. MP distal to A, not divided, semicircular, widely emarginated; FLP + A complex involved by MP.

\section{Material examined}

Lectotype (for Canthon speculifer var. subviridis Schmidt 1922; here designated) BRAZIL: O, Espir. Santo (white label) / 3271 E92+ (blue) / LECTOTYPE, Canthon speculifer var subviridis A. Schmidt, des F.Z. Vaz-de-Mello, 2014 (red with black margins) (NMRS 3271 E92+).

\section{Paralectotypes}

BRAZIL: 1 đ̊, Espir. Santo (white label) / 3271 E92+ (blue) / PARALECTOTYPE, Canthon speculifer var subviridis A. Schmidt, des F.Z. Vaz-de-Mello, 2014 (yellow with black margins) (NMRS 3271

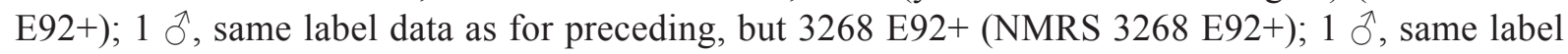
data as for preceding, but 3269 E92+ (NMRS $3269 \mathrm{E} 92+$ ); 1 ô, same label data as for preceding, but 3270 E92+ (NMRS 3270 E92+); 1 ô, same label data as for preceding, but 3285 E92+ (NMRS 3285 E92+); 1 o , same label data as for preceding, but 3277 E92+ (NMRS 3277 E92+); 1 \%, same label data as for preceding, but $3287 \mathrm{E} 92+$ (NMRS $3287 \mathrm{E} 92+)$; 1 \% , same label data as for preceding, but 3286 E92+ (NMRS3286 E92+); 1 + , same label data as for preceding, but 3288 E92+ (NMRS 3288 E92+).

Non-type material (68 specimens)

BRAZIL - Ceará: 1 + , C Pioson, coll. Hangay, Walter Heinz ex coll. and leg. (CEMT). - Alagoas: 1 đ, Ibateguara, 12 Oct. 2007, B.K.C. Filgueiras leg. (CEMT); 1 ô, Ibateguara, Serra Grande, 859'44" S, 3553'15" W, Mata Atlântica borda, pitfall, 1 Nov. 2011, B.K.C. Filgueiras leg. (CEMT). - Bahia: $3 \hat{\partial} \hat{\jmath}, 4$ 우, Itabuna, CEPLAC, Cabruca, 10 Jan. 2003, M. Santos leg. (CEMT). - Minas Gerais:

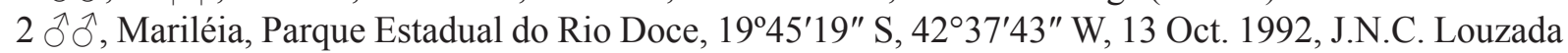
leg. (CEMT); 1 §, Mariléia, Pq. Est. Rio Doce, Feb. 2000, L. Scoss leg. (CEMT); 1 đ, same data as for preceding, but Nov. 1992, J.N.C. Louzada leg. (CEMT); 1 요 Ipatinga, Aug. 1994, G. Turrer leg. (CEMT). - Espírito Santo: $1 \overbrace{}^{\Uparrow}$, Soorotema, Reserva Natural Vale, Estrada Cinco Folhas, $19^{\circ} 08^{\prime 3} 34^{\prime \prime}$ S, 4003'56" W, 59 m a.s.1., human faeces, pitfall, 2 Feb. 2015, T. Vargas and L. Lopes leg. (CEMT); 1 万’, 1 ․ Soorotema, Reserva Natural Vale, Estrada Cinco Folhas, $19^{\circ} 08^{\prime} 56^{\prime \prime} \mathrm{S}, 40^{\circ} 04^{\prime} 08^{\prime \prime} \mathrm{W}, 71 \mathrm{~m}$ a.s.l., pitfall, 3 Feb. 2015, T. Vargas and L. Lopes leg. (CEMT); 2 우, Soorotema, Reserva Natural Vale, 
Estrada Cinco Folhas, $19^{\circ} 08^{\prime} 57^{\prime \prime}$ S, 4004'07" W, 65 m a.s.1., human faeces, pitfall, 3 Feb. 2015, T. Vargas and L. Lopes leg. (CEMT); 1 ', 1 , same data as for preceding, but $19^{\circ} 08^{\prime} 58^{\prime \prime} \mathrm{S}, 40^{\circ} 04^{\prime} 07^{\prime \prime} \mathrm{W}, 70 \mathrm{~m}$ a.s.1., (CEMT); 1 + , Soorotema, Reserva Natural Vale, Estrada Cinco Folhas, $19^{\circ} 08^{\prime} 32^{\prime \prime} \mathrm{S}, 40^{\circ} 03^{\prime} 55^{\prime \prime} \mathrm{W}$, 59 m a.s.1., human faeces, pitfall, 2 Feb. 2015, T. Vargas and L. Lopes leg. (CEMT); 1 $\uparrow, 2$ 우, same data as for preceding, but $19^{\circ} 09^{\prime} 09^{\prime \prime} \mathrm{S}, 40^{\circ} 02^{\prime} 19^{\prime \prime} \mathrm{W}$ (CEMT); 1 ô, Soorotema, Reserva Natural Vale, Estrada Flamengo, $19^{\circ} 09^{\prime} 07^{\prime \prime} \mathrm{S}, 40^{\circ} 03^{\prime} 00^{\prime \prime} \mathrm{W}, 11 \mathrm{~m}$ a.s.l., human faeces, pitfall, $2 \mathrm{Feb}$. 2015, T. Vargas and L. Lopes leg. (CEMT); 1 q, same data as for preceding, but $17 \mathrm{~m}$ a.s.1. (CEMT). - Rio de Janeiro:

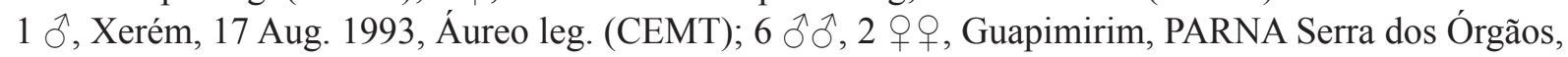
-22.530383, -43.001075, 100 m a.s.1., pitfall, 16-18 Dec. 2014, C. Araújo and R. Andrade leg. (CEMT);

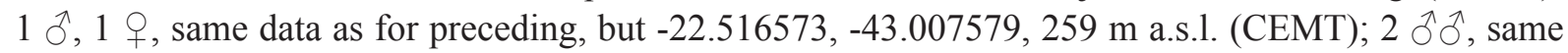
data as for preceding, but $-22.494686,-43.002185,400 \mathrm{~m}$ a.s.1. (CEMT); $1 \hat{\jmath}$, same data as for preceding, but $-22.426,-42.992918,600 \mathrm{~m}$ a.s.l. (CEMT); 2 우, same data as for preceding, but -22.524244 , $-43.000526,150 \mathrm{~m}$ a.s.1. (CEMT); 1 §, same data as for preceding, but $-22.468017,-42.998243,800 \mathrm{~m}$

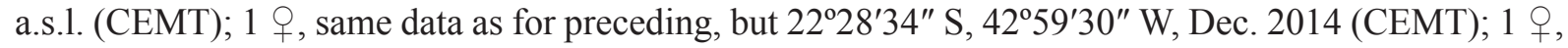

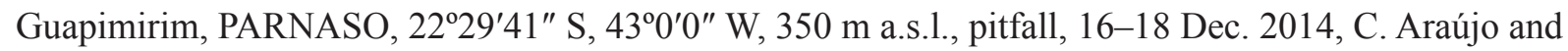
R. Andrade leg. (CEMT); 1 \%, same data as for preceding, but $22^{\circ} 31^{\prime} 05^{\prime \prime} \mathrm{S}, 43^{\circ} 0^{\prime} 17^{\prime \prime} \mathrm{W}, 200 \mathrm{~m}$ a.s.1.

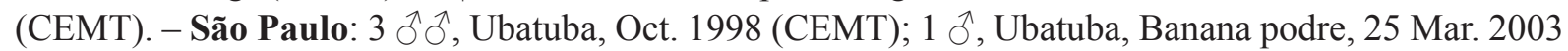

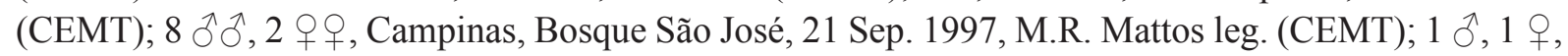
same data as for preceding, but 19 Aug. 1997, M. Castro leg. (CEMT).

\section{Distribution}

Brazilian Atlantic Forest in the states of Alagoas, Bahia, Ceará, Espírito Santo, Minas Gerais and Rio de Janeiro (Fig. 8).

\section{Taxonomic remarks}

Canthon smaragdulus subviridis stat. rev. was described as a variety of C. speculifer (Schmidt 1922). It was also listed as a variety of Goniocanthon smaragdulus in a catalogue, but Vulcano \& Pereira (1964) did not explicitly treat this as a taxonomic act, nor did they consult the type material. Finally, this taxon is also treated as a variety of C. smaragdulus in a catalogue by Vaz-de-Mello (2000).

Canthon (Goniocanthon) fulgidus Redtenbacher, 1868

Figs 1D-F, I-J, 3, 7-8, 10

\section{Redescription (male)}

CoLour. Dorsal surface with golden metallic sheen.

HEAD. Frontal surface smooth, lacking horns or tubercles. Clypeal teeth with rounded apex, with thin setae at apex and separated by a U-shaped emargination. Clypeo-genal junction slightly emarginated, rounded. Clypeo-genal suture present.

Pronotum. Disc simply convex, smooth, lacking microscultpure $(120 \times$ magnification $)$. Anterior and posterior angles acute. Lateral borders effaced. Posterior border with lateral semicircular emarginations near to posterior angles.

HYPOMERON. Lacking transverse keel, surface black with chagrined microsculpture. Sparse setae present at anterior portion.

Metasternum. Anterior lobe slightly convex (Fig. 1E). 
ELYTRA. Striae weakly impressed, more distinct near elytral basis, $8^{\text {th }}$ stria carinate along its basal third. Interstriae flat.

LEGS. Protibiae three-toothed, dorsal surface with two tufts of setae present on each tibial tooth. Protibial spur truncated, wider than long and apically emarginated, forming two spines. External border of protibia serrated. Profemur ventral surface black, glabrous, with a distal tooth on its anterior border. Ventral face of meso- and metatibiae rugose on posterior half (Fig. 1D). Meso- and meta-trochanter with a hair tuft near femur articulation.

AвDOMEN. Ventrites glabrous, lacking punctures and not narrowed medially.

Pygidium. Convex. Basal line delimiting pygidium from pro-pygidium. Metallic sheen pattern similar to those of pronotal disc.

Paramera. In lateral view (Fig. 1I): flattened; apex truncated; upper angle rounded; lower angle acute, forming a spine or tooth. On dorsal view (Fig. 1J): membranous portion wider at central portion and narrower near apex and basis. Apex rounded.

INTERNAL SAC (Fig. 7). FLP involving A forming a central duct with spiniform apex. SA lateral to FLP + A complex. MP distal to A, semicircular, widely emarginated; FLP + A complex involved by MP. SRP with lateral prolongation.

\section{Morphological variation}

BL: 8-16 mm, PW: 5-10 mm. Dorsal surface metallic sheen may vary between green, golden or red metallic colouration (Fig. 3A-C). Protibial spur of females as wide as long, with apical U-shaped emargination. Pygidium and metasternal anterior lobe less protuberant in females than in males.

Canthon (Goniocanthon) fulgidus fulgidus Redtenbacher, 1868

Figs 1D-F, I-J, 3A, 8, 10

Canthon fulgidus Redtenbacher, 1868: 51.

Canthon fulgidus - Gemminger \& Harold 1869: 991 (catalogue). — Gillet 1911: 29 (catalogue). Schmidt 1920: 118 (taxonomy); 1922: 69 (key). — Balthasar 1939: 203 (key); 1941: 342 (catalogue); 1951: 327 (catalogue). — Blackwelder 1944: 199 (catalogue). — Guérin 1953: 257 (catalogue). Goniocanthon fulgidus - Pereira \& Martínez, 1956: 111. — Vulcano \& Pereira 1964: 593 (catalogue). Canthon (Goniocanthon) fulgidus - Halffter \& Martínez, 1977: 75. — Vaz-de-Mello 2000: 191 (species list).

\section{Diagnosis}

In the subgenus, $C$. (G.) fulgidus fulgidus is separated by the following combination of characters: mesoand metafemora ventral surface rugose on its posterior half (Fig. 1D); dorsal surface of pronotum and elytral disc with similar golden metallic colouration (Fig. 3A); metasternum anterior lobe weakly convex (Fig. 1E); profemur with a tooth on its anterior border. Paramera in lateral view (Fig. 1I): flattened; apex truncated; upper angle rounded; lower angle acute, forming a spine or tooth. Internal sac (identical to Canthon (G.) fulgidus martinezi subsp. nov., see Fig. 7): FLP involving A forming a central duct with spiniform apex. SA lateral to FLP + A complex present. MP distal to A, not divided, semicircular, widely emarginated; FLP + A complex involved by MP. 


\section{Material examined}

Lectotype (for Canthon fulgidus Redtenbacher, 1868; here designated)

BRAZIL: Ō, Natt 12 (brown label) / C. fulgidus det. Letraf 93 (white) / TYPUS (red) / fulgidus Redt (white) / fulgidus Brasil Redt. (white with black margins) / LECTOTYPE, Canthon fulgidus Redtenb., des F.Z. Vaz-de-Mello 2013 (NMW).
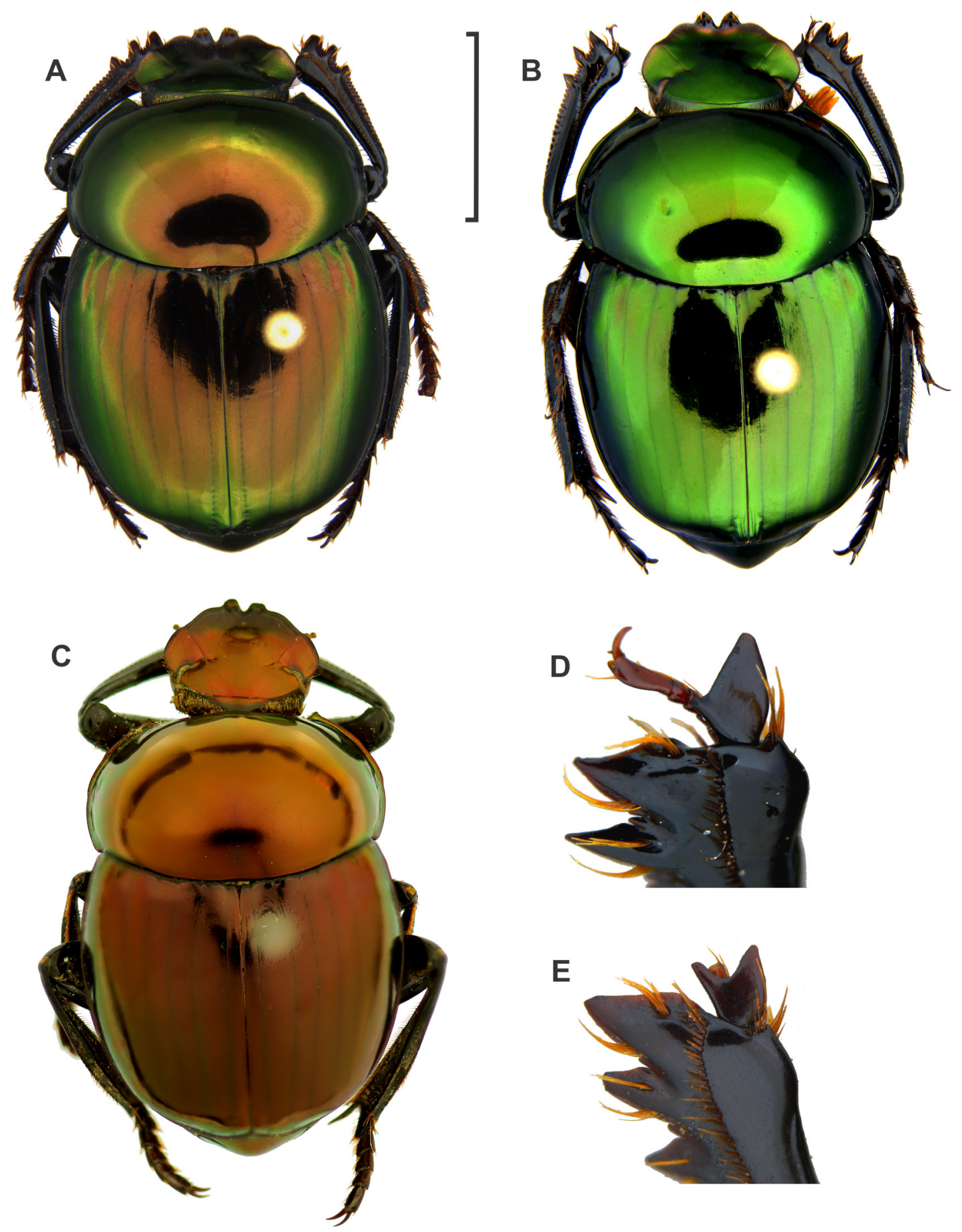

Fig. 3. A. Canthon (G.) fulgidus fulgidus Redtenbacher, 1868. B. C. (G.) fulgidus martinezi subsp. nov. C-E. C. (G.) fulgidus pereirai subsp. nov. D. Male protibial spur. E. Female protibial spur. Scale bar: $\mathrm{A}-\mathrm{C}=5.0 \mathrm{~mm}$. 
Non-type material (106 specimens)

BRAZIL - Mato Grosso: 1 +, Apiacás, Boca da Onça, Varredura, Jan.-May 2008, N. Pinho leg. (CEMT); 1 ô, Alta Floresta, Chácara Recanto das Orquídeas, 956'14" S, 56 ${ }^{\circ} 7^{\prime} 12^{\prime \prime}$ W, 2 Apr. 2009,

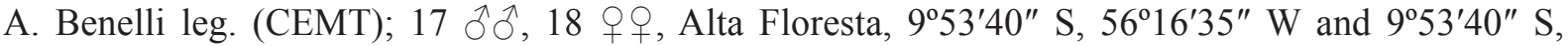
$56^{\circ} 16^{\prime} 34^{\prime \prime} \mathrm{W}$, frag. 26 centro, human faeces, pitfall, Jun. 2008, E. Berenguer leg. (CEMT); 1 ㄱ, 2 우, Alta Floresta, 9 $47^{\prime} 56^{\prime \prime}$ S, 55 55'51" W, frag. 62, human faeces, pitfall, May 2008, E. Berenguer leg.

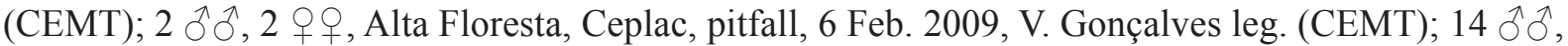
3 우, same data as for preceding, but 24 May 2009 (CEMT); 1 , same data as for preceding, but

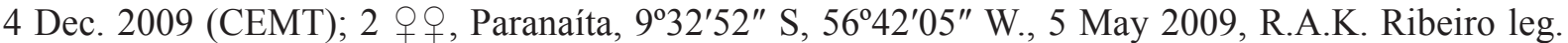

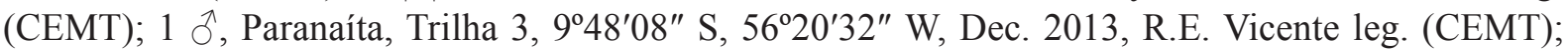

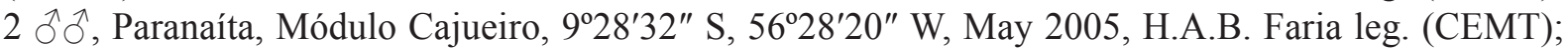

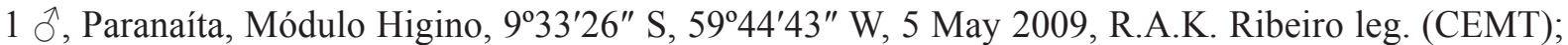
1 ๙ , 2 우, Cláudia, $11^{\circ} 25^{\prime} 16^{\prime \prime} \mathrm{S}, 5^{\circ} 18^{\prime} 35^{\prime \prime} \mathrm{W}$, PPBIO-II T1P3, $320 \mathrm{~m}$ a.s.1., mistnet 1-1.5 $\mathrm{m}$ a.s.1.,

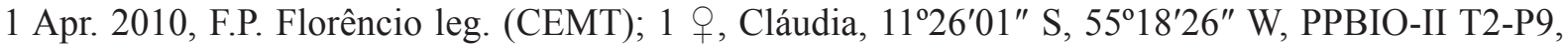

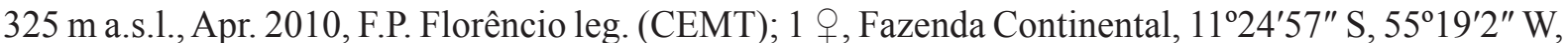
20 Feb. 2010, M.F. Souza leg. (CEMT); 1 q, same data as for preceding, but, $11^{\circ} 35^{\prime} 59^{\prime \prime} \mathrm{S}, 55^{\circ} 15^{\prime} 55^{\prime \prime} \mathrm{W}$

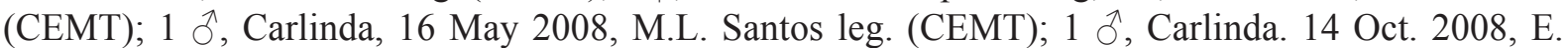
Macena leg. (CEMT); 1 \& Carlinda, 15 Oct. 2008, N. Gavilan leg. (CEMT); 1 ô, Carlinda, 16 Oct.

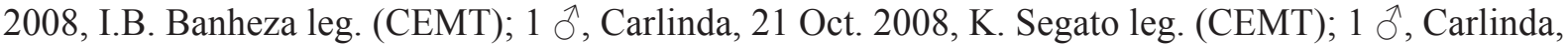

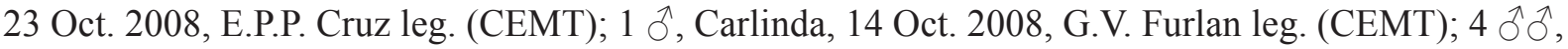
1 ㅇ, Carlinda, 10 Oct. 2009, E. Teles leg. (CEMT); 1 ภ, Carlinda, 11 Oct. 2009, M.M.C. Pril leg.

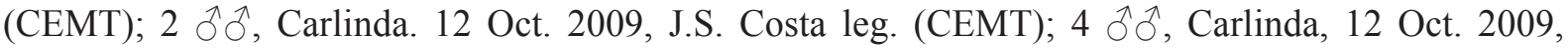
V. Nunes leg. (CEMT); 1 क , Carlinda, 17 Oct. 2009, L.C. Santos leg. (CEMT); 1 ô, Carlinda, 18 Oct.

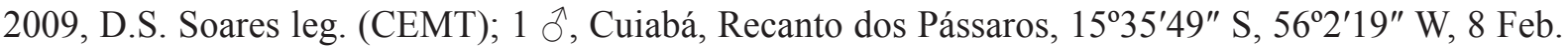
2009, A.K. Azevedo leg. (CEMT).

\section{Distribution}

Brazil, state of Mato Grosso, covering Amazon-Cerrado transitions including semi-deciduous and gallery forests (Fig. 8).

\section{Natural history remarks}

This subspecies and C. fulgidus pereirai subsp. nov. (described later in this paper) are found in Amazonian primary and secondary forests as well as in semideciduous and gallery forests in the states of Mato Grosso and Rondônia. However, both subspecies are especially abundant in várzea forest environments, where individuals of $C$. fulgidus are easily found perching on larger leaves exposed to sunlight in forest understory between $0.50 \mathrm{~m}$ and $2.5 \mathrm{~m}$ high (Fig. 10). Both males and females can wait up to 30 minutes on the same leaf, usually in the warmer hours of the day (between 10 am and $4 \mathrm{pm}$ ), only moving the antennae, probably trying to capture some odoriferous plume as described in Louzada (1998) for similar species. We have observed several times that, after perching, the individuals fly in the direction of some kind of primate faeces, including human. When manipulated, individuals of both subspecies release an odour of rotten or fermented fruit.

Canthon (Goniocanthon) fulgidus martinezi subsp. nov. urn:1sid:zoobank.org:act:09023BB9-E175-4267-A289-A48D682ABBC2

Figs 3B, 7-8

\section{Diagnosis}

In the subgenus, $C$. (G.) fulgidus martinezi subsp. nov. is separated by the following combination of characters: meso- and metafemora ventral surface rugose on its posterior half (Fig. 1D); dorsal surface 
NUNES L.G. DE O.A. et al., Revision of the South American subgenus Canthon (Goniocanthon)

of pronotum and elytral disc with similar metallic green metallic colouration (Fig. 3B); metasternal anterior lobe slightly convex (Fig. 1E); profemur with a tooth on its anterior border. Paramera in lateral view (Fig. 1I): flattened; apex truncated; upper angle rounded; lower angle acute, forming a spine or tooth. Internal sac (Fig. 7): FLP involving A forming a central duct with spiniform apex. SA lateral to FLP + A complex present. MP distal to A, not divided, semicircular, widely emarginated; FLP + A complex involved by MP.

\section{Etymology}

The specific epithet refers to Antônio Martínez (1922-1993), Argentinean taxonomist, author of Goniocanthon and of several other taxa of Scarabaeinae.

\section{Material examined}

\section{Holotype}

ECUADOR: $\widehat{\jmath}$, Francisco de Orellana, Rodrigo Borja, IAMOE, 0 $41^{\prime} 50^{\prime \prime} \mathrm{S}, 7^{\circ} 8^{\prime 2} 25^{\prime \prime} \mathrm{W}$, human faeces, pitfall, Jun. 2000, Dávalos leg. (CEMT).

Paratypes (49 specimens)

COLOMBIA: 1 ô, Guaviare, S.J. Del Guaviare, Fianca Eli, 2²1'46.2" N, 72³8'29.9” W, 200 m a.s.1., faeces of macaco, Jan. 2008, M.C. Santos leg. (CEUN).

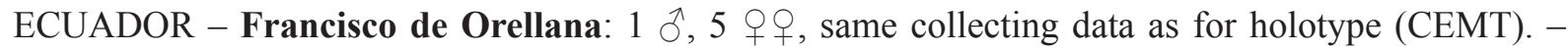
Orellana (Napo): 2 우, PUCE Yasuni, $0^{\circ} 40^{\prime} 00^{\prime \prime} \mathrm{S}, 76^{\circ} 23^{\prime} 00^{\prime \prime} \mathrm{W}, 250 \mathrm{~m}$ a.s.l., primary forest, human faeces, pitfall, Jun. 1995, M. Bass and N. Pitfan leg. (CEMT); 1 + , Parque Nacional Yasuni, $0^{\circ} 56^{\prime} 00^{\prime \prime}$ S,

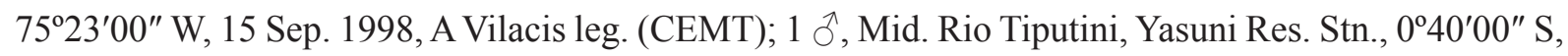
$76^{\circ} 24^{\prime} 00^{\prime \prime} \mathrm{W}$, flight intercept trap, Jul. 1999, A. Tishechkin leg. (CEMT); 1 \&, Yasuni National Park, $0^{\circ} 38^{\prime} 00^{\prime \prime}$ S, 76 ${ }^{\circ} 36^{\prime} 00^{\prime \prime}$ W, 215 m a.s.l., lowland rainforest, 8 Jan. 1998, Ratcliffe, Jameson, Smith and Villatoro leg. (CEMT).

PERU: 1 q, Loreto, Rio Pucacuru, 0120668 E, 9761668 N, pitfall, carrion, 1 Dec. 2007, C. Moreno leg. (CEMT); 1 §, Junin, Satipo, Gloribamba, Jan. 2004, H. Rojas leg. (CEMT).

BRAZIL - Amazonas: 8 $\widehat{\jmath}, 7$ + , Carauari, Reserva Extrativista do Médio Juruá, $5^{\circ} 31^{\prime} 17^{\prime \prime}$ S,

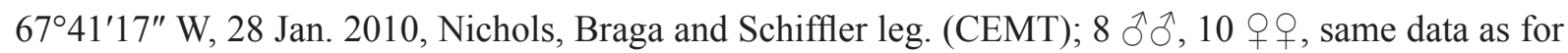
preceding, but 5 $31^{\prime} 20^{\prime \prime} \mathrm{S}, 67^{\circ} 41^{\prime} 20^{\prime \prime} \mathrm{W}$ (CEMT).

BOLIVIA: $1{ }^{\AA}, 1$ q, Pando, Santa Rosa, Manuripi National Wildlife Reserve, 1200' S, 68 $52^{\prime}$ W, $190 \mathrm{~m}$ a.s.1., 24 Oct. 2004. A.C. Hamel and D. Aguirre leg. (CEMT).

\section{Distribution}

Bolivia (Pando), Brazil (Amazonas), Colombia, Ecuador and Peru (Fig. 8).

\section{Taxonomic remarks}

This subspecies is cited for Colombia (Medina et al. 2001) and Peru (Ratcliffe et al. 2015) as Canthon (Goniocanthon) smaragdulus because of its green metallic sheen, which allows it to be confused with the northern subspecies $C$. (G.) smaragdulus subviridis stat. rev. (diagnosed above). This subspecies is cited as Canthon fulgidus for Satipo province, Peru (Balthasar 1951). 
Canthon (Goniocanthon) fulgidus pereirai subsp. nov. urn:lsid:zoobank.org:act:AD1C82C4-4976-4FBC-96DC-0980C27C1922

Fig. 3C-E, 8

\section{Diagnosis}

In the subgenus, $C$. (G.) fulgidus pereirai subsp. nov. is separated by the following combination of characters: meso- and metafemora ventral surface rugose on posterior half (Fig. 1D); dorsal surface of pronotum and elytral disc with similar metallic red colouration (Fig. 3C); metasternal anterior lobe slightly convex (Fig. 1E); profemur with a tooth on its anterior border. Paramera in lateral view (Fig. 1I): flattened; apex truncated; upper angle rounded; lower angle acute, forming a spine or tooth. Internal sac (identical to Canthon (G.) fulgidus martinezi subsp. nov., see Fig. 7): FLP involving A forming a central duct with spiniform apex. SA lateral to FLP + A complex present. MP distal to A, not divided, semicircular, widely emarginated; FLP + A complex involved by MP.

\section{Etymology}

The specific epithet refers to Francisco Silvério Pereira (1913-1991), priest, taxonomist, author of Goniocanthon and of several other taxa of Scarabaeinae.

\section{Material examined}

Holotype

BRAZIL: Õ Pará, Redenção, Faz. Marajoara, 0749.741’ S, 50¹5.910’ W, 13 Oct. 1998, P. Scheffler leg. (CEMT).

Paratypes (141 specimens)

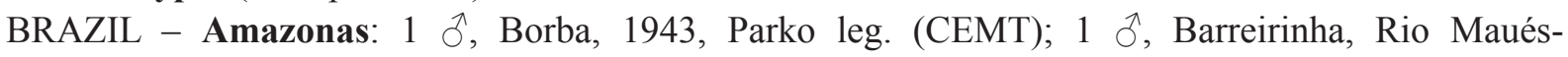

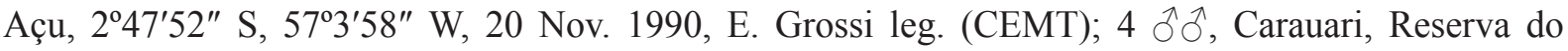
Desenvolvimento Sustentável do Uacari, 546'6" S, 67 42'40" W, 4 Aug. 2009, Nichols, Braga and

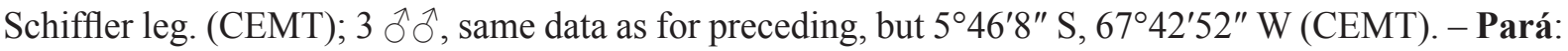

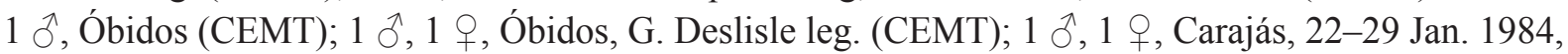
N. Degallier leg. (CEMT); 8 $\widehat{\jmath}$, Alter do Chão, RESEX Tapajós Arapiuns, FIT, 22 Dec. 1998 (CEMT);

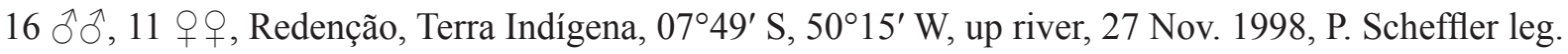

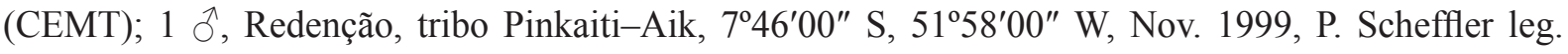
(CEMT); 1 q, Redenção, Fazenda Marajoara, $7^{\circ} 50^{\prime} 00^{\prime \prime}$ S, 50¹6'00" W, Oct. 1998, P. Scheffler leg.

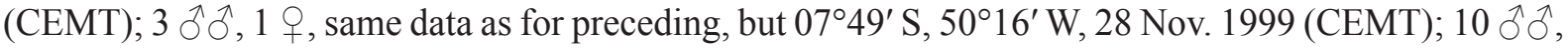
11 q $q$, same data as for holotype (CEMT); $14 \widehat{\jmath} \widehat{\jmath}, 15$ q $q$, same data as for holotype, but 1 Dec. 1999

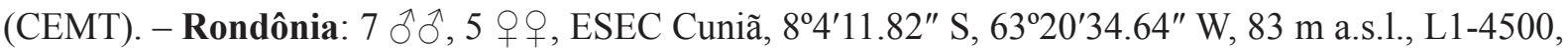

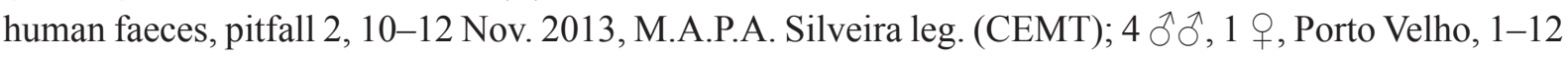
Jun. 2012, R.M. Moraes leg. (CEMT); 1 §ૈ, 1 q, Porto Velho, Jun. 2011, R.V. Nunes and M.F. Souza

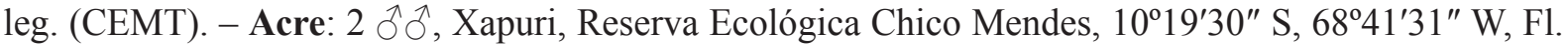

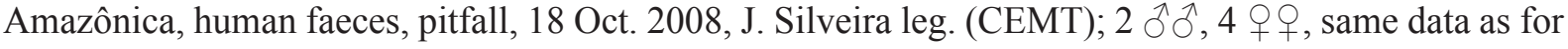
preceding, but 10²4'39" S, 68 59'55" W, 17 Oct. 2008, R. Andrade leg. (CEMT). - Mato Grosso: 1 q, Cotriguaçú, Fazenda São Nicolau, 951'22" S, 58¹5'11" W, 14 Dec. 2009, PROA Correa leg. (CEMT);

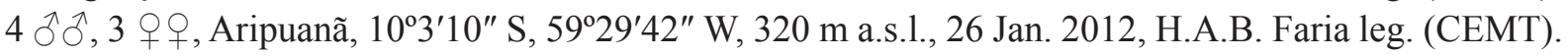

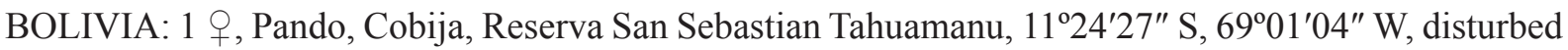
Amazon forest, day collected, along forest track, 20 Dec. 2003, D.J. Mann and A.C. Hamel leg. (OUMNH). 
NUNES L.G. DE O.A. et al., Revision of the South American subgenus Canthon (Goniocanthon)

\section{Distribution}

Bolivia (Pando), Brazil (Acre, Amazonas, Mato Grosso, Pará and Rondônia) (Fig. 8).

Canthon (Goniocanthon) bicolor Castelnau, 1840

Figs 4, 8

Canthon bicolor Castelnau, 1840: 69.

Canthon bicolor - Harold 1868: 14, 71 (key). — Gemminger \& Harold 1869: 990 (catalogue). — Gillet 1911: 28 (catalogue). — Schmidt 1922: 64, 73 (key). — Balthasar 1939: 189 (key). — Blackwelder 1944: 198 (catalogue). - Vulcano \& Pereira 1964: 604 (catalogue). - Halffter \& Martínez 1977: 39, 70 (taxonomic remarks). — Vaz-de-Mello 2000: 191 (species list).

\section{Diagnosis}

In the subgenus, $C$. (G.) bicolor Castelnau, 1840 is separated by the following combination of characters: ventral surface of meso- and metafemora smooth (Fig. 4B); dorsal surface of pronotum and elytral disc with different colours, pronotum with light green metallic sheen and elytra with dark green metallic sheen (Fig. 4A). Metasternal anterior lobe flat (Fig. 4B). Profemur without teeth on anterior margin. Paramera, in lateral view (Fig. 4F): flattened; apex semicircular; upper angle rounded; lower angle triangular. In dorsal view (Fig. 4G). Internal sac (Fig. 9): FLP not completely involving A forming two duct with spiniform apex. SA lateral to FLP + A complex absent. MP distal to A, divided in MP 1 and MP 2, MP 1 semicircular, MP 2 fishhook-shaped with two hook; FLP + A complex involved by MP 1.

\section{Material examined}

Lectotype (for Canthon bicolor Castelnau, 1840; here designated)

FRENCH GUIANA: 9 , Coprobius Bicolor DeLaporte anim. art. p. 69 Canthon $\mathrm{n}^{\circ} 6$ Cayennae Maroni D'DeLaporte (rectangular green label, border black) / $G$ (small red label) / A. Gory coll. purchased 1849-1850 From H.L.GORY (white label) standing over: Coprobius bicolor DeLaporte, Cayennae OX. UNI. MUS. NAT. HIST. (OUMNH) (white label) / LECTOTYPE $q$ Canthon bicolor Cast. des. F.Z. Vaz-de-Mello, 2013" (red label) (OUMNH).

Non-type material (248 specimens)

VENEZUELA - Bolívar: 3 q 9 , Altiplanicie de Nuria ("El Hormiguero, Meseta de Nuria"), 500 m a.s.l., Expediction Instituto Zoologia Agricola de la Faculd de Agronomia, 13-17 Dec. 1974, Universidad Central de Venezuela leg. (CMNC); 2 우, Sinfontes, $22 \mathrm{~km} \mathrm{~S}$ of El Dorado, flight interception trap, 25 Jun.-12 Jul. 1987, S. and J. Peck leg. (CMNC); 1 क , Sinfontes, 33 km S of El Dorado, $220 \mathrm{~m}$ a.s.l., 2-7 Aug. 1986, B. Gill leg. (CMNC); 1 क, Sinfontes, El Bochinche, Reserva Florestal Imataca, $200 \mathrm{~m}$ a.s.1., Expediction Instituto Zoologia Agricola de la Faculd de Agronomia, 6-13 Dec. 1974, Universidad Central de Venezuela leg. (CMNC).

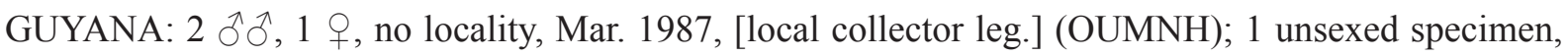
Essequibo River, Monkey Jump, 19 Aug. 1929, Oxford University Expedition to British Guiana leg. $(\mathrm{BMNH})$.

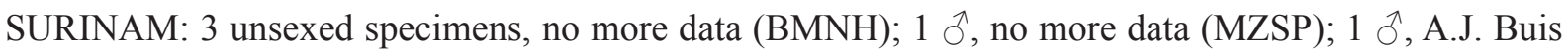
and V. Balthasar leg. (NMPC); 1 , no locality or date, V. Balthasar leg. (NMPC); 1 , no locality and date, Dr. Will leg. (ZMHB). - Paramaribo: 2 $\widehat{\jmath}, 1$, , no locality or date, S.V. Heller leg. (ZMHB). -

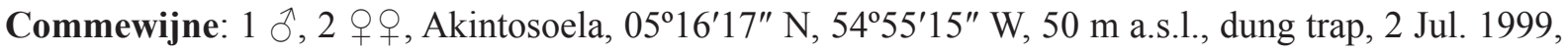
Z. Falin leg. (CMNC); 1 q, Akintosoela, $05^{\circ} 16^{\prime} 17^{\prime \prime} \mathrm{N}, 54^{\circ} 55^{\prime} 15^{\prime \prime} \mathrm{W}, 40 \mathrm{~m}$ a.s.l., flight interception 
trap, 3 Jul. 1999, Z. Falin leg. (CMNC). - Marowijne: 1 क, Palumeu, 0320'56" N, 55²6'18" W, flight interception trap, 9 Jul. 1999, Z. Falin leg. (CMNC).

FRENCH GUIANA: 1 unsexed specimen, no locality, 1981, G. Tavakilian leg. (MNHN); 1 q, "D-5 4k SE Tngmd Jct", 24 Aug. 1995, J.E. Wappes leg. (CMNC); 1 ㅇ, PK 45, Piste del Beligan, 7 Mar.

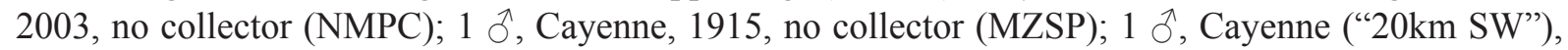
04 $48^{\prime} 18^{\prime \prime} \mathrm{N}, 52^{\circ} 28^{\prime} 41^{\prime \prime} \mathrm{W}, 30$ m a.s.l., flight interception trap, 29 May-9 Jun. 1997, J. Ashe and R. Brooks leg. (CMNC); 1 đ̃, 1 , Cayenne, Camopi, Oyapock river, Ilet Massikiri, [dung], 17 Nov. 1969,

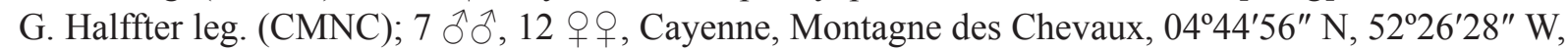
$75 \mathrm{~m}$ a.s.1., 25 Sep. 2011, SEAG leg. (CEMT); 4 के $\hat{0}, 1$ ㅇ, same data as for preceding, but 27 Jan.

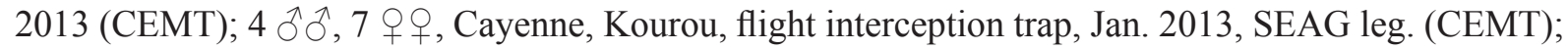
3 ภึ, 1 q, Cayenne, La Chaumière, Apr. 1978, P. Arnaud leg. (CMNC); 2 + , Cayenne, [Réserve naturelle nationale des] Nouragues, $04^{\circ} 05^{\prime}$ N, 52 $40^{\prime}$ W, 155 m a.s.l., Mar. 1997, F. Feer leg. (CEMT); $3 \hat{\delta} \hat{\delta}, 1$, same data as for preceding, but May 2003 (CEMT); $5 \hat{\delta} \hat{\delta}, 4$ q $q$, Cayenne, Paracou

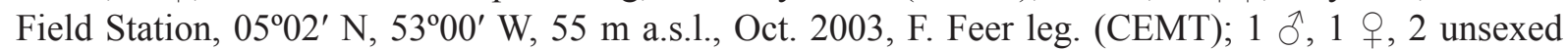

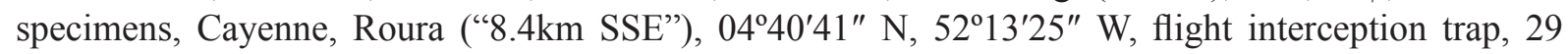
May-10 Jun. 1997, J. Ashe and R. Brooks leg. (CMNC); 2 우, Cayenne, Roura, Montagne de Kaw,

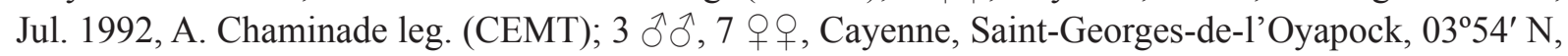
$51^{\circ} 48^{\prime}$ W, 35 m a.s.1., May 2014, F. Feer leg. (CEMT); 4 unsexed specimens, Nouveau Chantier, no date or collector (MNHN - Le Moult collection); 1 §, Nouveau Chantier, no date or collector (ZMHB - Le Moult collection); 1 specimen, Nouveau Chantier, Bas-Maroni, no date or collector (MNHN); 6 ડ̄ $^{\lambda}, 12$ 우, 3 unsexed specimens, Régina ("S of Régina”), 30 Dec. 2006, M. Snižek leg. (NMPC);

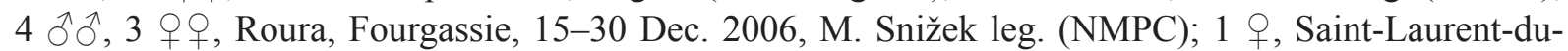
Maroni, no date and collector (ZMHB - Le Moult collection); 1 unsexed specimen, Saint-Laurentdu-Maroni ("Maroni"), no date or collector (MNHN); 1 , , Saint-Laurent-du-Maroni ("Maroni”), no date or collector (ZMHB); 1 ô, 6 우, Saint-Laurent-du-Maroni ("Bas-Maroni"), no date or collector (ISNB - Le Moult collection); 1 unsexed specimen, Saint-Laurent-du-Maroni, "Jun", no collector (ZMHB); 1 §ै, Saint-Laurent-du-Maroni ("Maroni”), 1915, no collector (CMNC); 1 \%, Saint-Laurentdu-Maroni ("Maroni”), Set. 1925, no collector (MZSP); 1 đo, 1 क, Saint-Laurent-du-Maroni, Apr. 1976, F. Chalumeau leg. (CEMT); $2 \widehat{\partial} \widehat{\partial}, 1$ q, Saint-Laurent-du-Maroni, Apr. 1976, P. Arnaud leg. (CEMT);

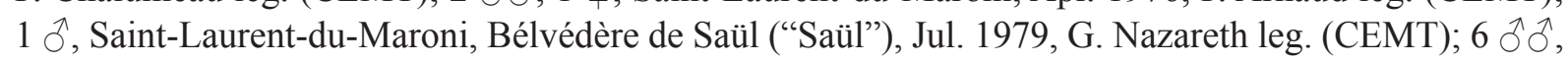

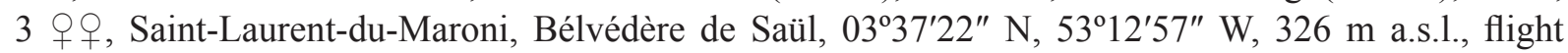
interception trap, 13 May 2011, SEAG leg. (CEMT); 7 $\widehat{0}, 3$ 9 우, Saint-Laurent-du-Maroni, Bélvédère

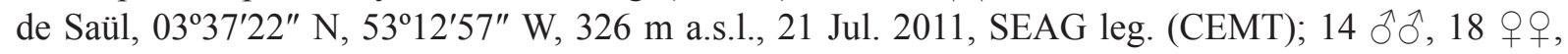
2 unsexed specimens, Saint-Laurent-du-Maroni ("20km E of St. Laurent du Maroni”), [route] Crique Naï, 10 Dec. 2006, M. Snižek leg. (NMPC); 1 ภ̂, 1 क , Saint-Laurent-du-Maroni, Maripasoula, Saül

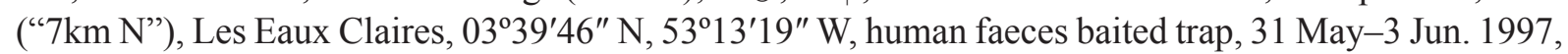
J. Ashe and R. Brooks leg. (CMNC); 1 ô, Saint-Laurent-du-Maroni, Maripasoula, Saül ("7km N"), Les

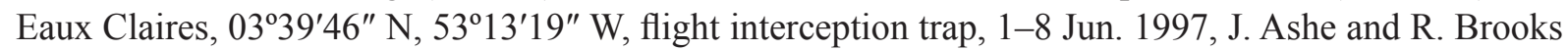
leg. (CMNC); 2 วิ ô, Saint-Laurent-du-Maroni, Maripasoula, Saül (“7km N"), Les Eaux Claires, Mount

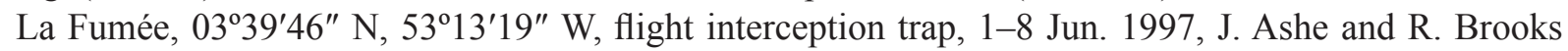
leg. (CMNC); 1 q, Saint-Laurent-du-Maroni, Maripasoula, Saül ("7km N"), Les Eaux Claires, Mount

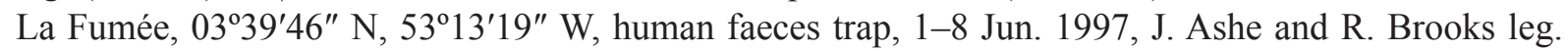
(CMNC); 3 우, Saint-Laurent-du-Maroni, Maripasoula, Saül ("7km N"), Les Eaux Claires, Mount

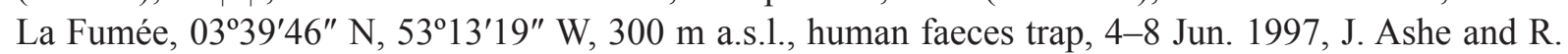
Brooks leg. (CMNC); $2 \widehat{\partial}^{\jmath}$, Saint-Laurent-du-Maroni, Maripasoula, Saül, Mount Galbao, $03^{\circ} 37^{\prime} 18^{\prime \prime}$ N, $53^{\circ} 16^{\prime} 42^{\prime \prime}$ W, 740 m a.s.l., flight interception trap, 5-7 Jun. 1997, J. Ashe and R. Brooks leg. (CMNC);

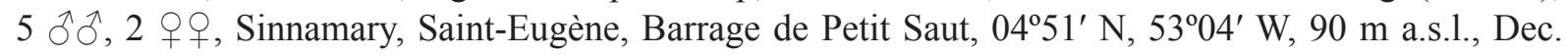
1998, F. Feer leg. (CEMT). 
BRAZIL: 1 unsexed specimen, no more data (MNHN); 1 , , illegible information (collector?) (ZMHB). - Amapá: 1 đ̂, 1 q, Serra do Navio, Jan. 1957, Pereira \& Martínez leg. (CMNC); 4 + , , Serra do Navio, Sep. 1957, Pereira \& Martínez leg. (CMNC); 1 ô, 2 unsexed specimens, Serra do Navio, 00 $53^{\circ} 06^{\prime \prime}$ N, 51 ${ }^{\circ} 52^{\prime} 53^{\prime \prime}$ W, Sep. 2000, R. Ribon leg. (CEMT). - Pará: 6 unsexed specimens, Almeirim, Monte Dourado, Pacanari, $0^{\circ} 81^{\prime}$ S, 52 56' W, Mar. 2006, T. Gardner and M. Hernández leg. (BMNH); 1 đ’,

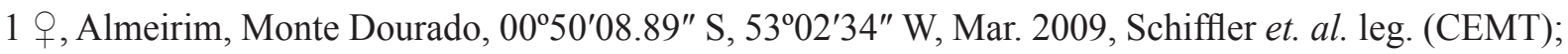
3 unsexed specimens, Santarém, Mar. 1990, O. Schmitt leg. (MNHN).

\section{Remarks on the lectotype designation}

The specimen designated herein as lectotype has a label with Pierre François Marie Auguste Dejean's handwriting indicating that François Louis Nompar de Caumont Laporte, the Comte of Castelnau, is the author of bicolor and the collector of that specimen. We are aware that Castelnau sold or donated his collections, but there is no evidence to state that all syntypes of Castelnau were sold to a single collection or went to a single place.

\section{Redescription}

MALE. Dorsal surface pronotum and elytra disc with different colours, pronotum with light green metallic sheen and elytra with dark green metallic sheen (Fig. 4A).

Head. Frontal surface smooth, lacking horns or tubercles. Clypeal teeth with rounded apex, with thin setae on apex and separated by a U-shaped emargination. Clypeo-genal junction slightly emarginated, rounded. Clypeo-genal suture present.

Pronotum. Disc simply convex, smooth, lacking microscultpure $(120 \times$ magnification). Anterior and posterior angles acute. Lateral borders effaced. Posterior border with lateral semicircular emarginations near to posterior angles.

HYPOMERON. Lacking transverse keel, surface black with chagrined microsculpture. Sparse setae present at anterior portion.

Metasternum. Metasternal anterior lobe flat (Fig. 4B).

ELYTRA. Striae weakly impressed, more distinct near elytral basis, $8^{\text {th }}$ stria carinate along its basal third. Interstriae flat.

Legs. Protibiae three-toothed, dorsal face with two tufts of setae present on each tibial teeth. Protibial spur truncated, larger than tall and apically emarginated, forming two spines (Fig. 4D). External border of protibia serrated. Profemur ventral face brown, glabrous, without a distal tooth on its anterior border. Ventral face of meso- and metatibiae rugose on posterior half (Fig. 4B). Meta-trochanter having a hair tuft near femur articulation.

АвDомen. Ventrites glabrous, lacking punctures and not narrowed medially.

Pygidium. Convex. Basal line delimiting pygidium from pro-pygidium. Metallic sheen pattern similar to that of pronotal disc.

PARAmera. In lateral view (Fig. 4F): flattened; apex semicircular; upper angle rounded; lower angle triangular. In dorsal view (Fig. 4G): membranous portion wider at central portion and narrower near apex and basis. Apex rounded. 


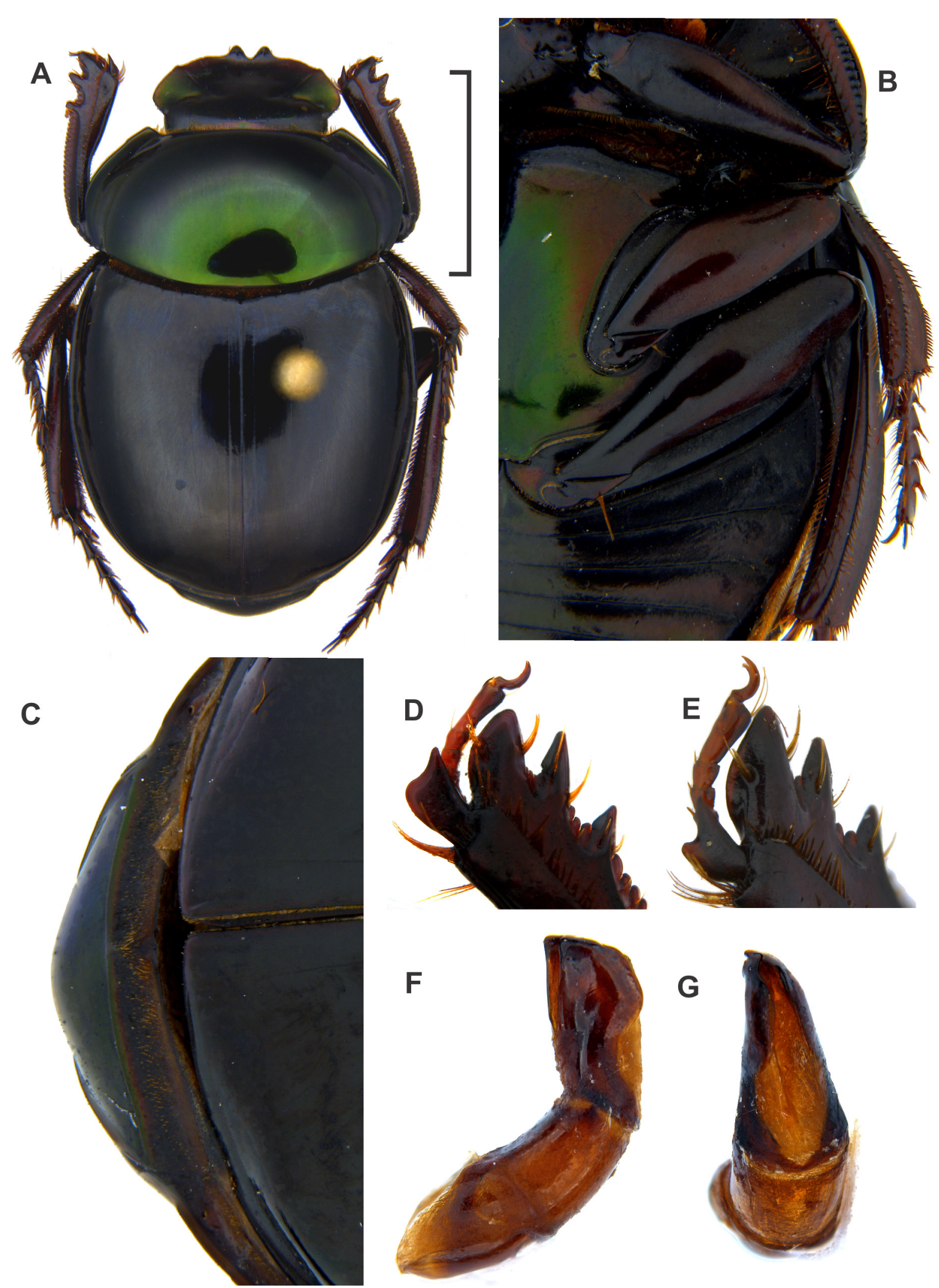

Fig. 4. Canthon (G.) bicolor Castelnau, 1840. A. Habitus, dorsal view. B. Ventral surface of mesoand metafemora and metasternal anterior lobe not convex. C. Dorsal view showing convex pygidium. D. Male protibial spur. E. Female protibial spur. F. Paramera lateral view. G. Paramera dorsal view. Scale bar: $\mathrm{A}=5.0 \mathrm{~mm}$. 

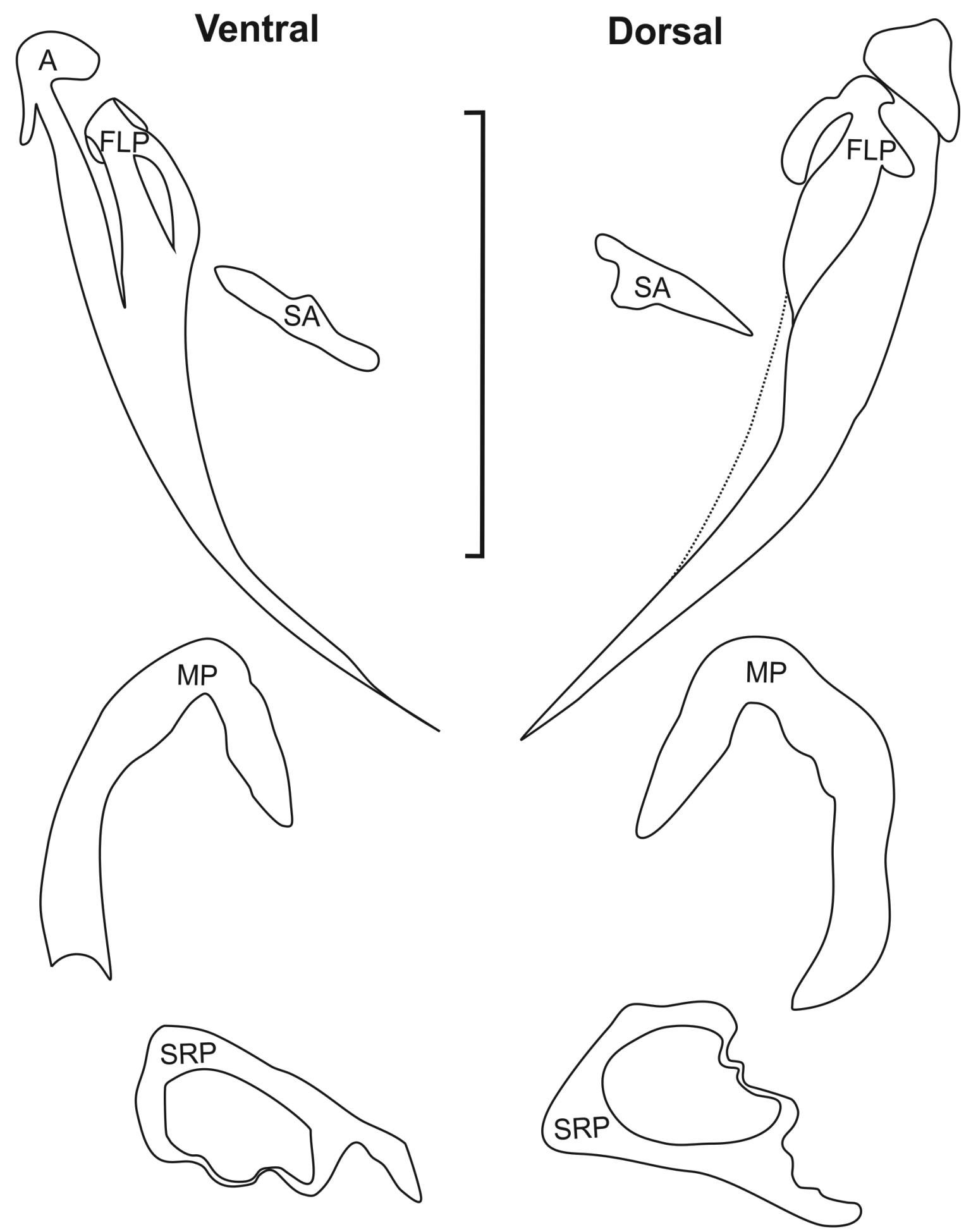

Fig. 5. Sclerites of the internal sac of Canthon (G.) smaragdulus subviridis Schmidt, 1922 stat. rev. Abbreviations: see Material and methods. Scale bar: $1.0 \mathrm{~mm}$. 


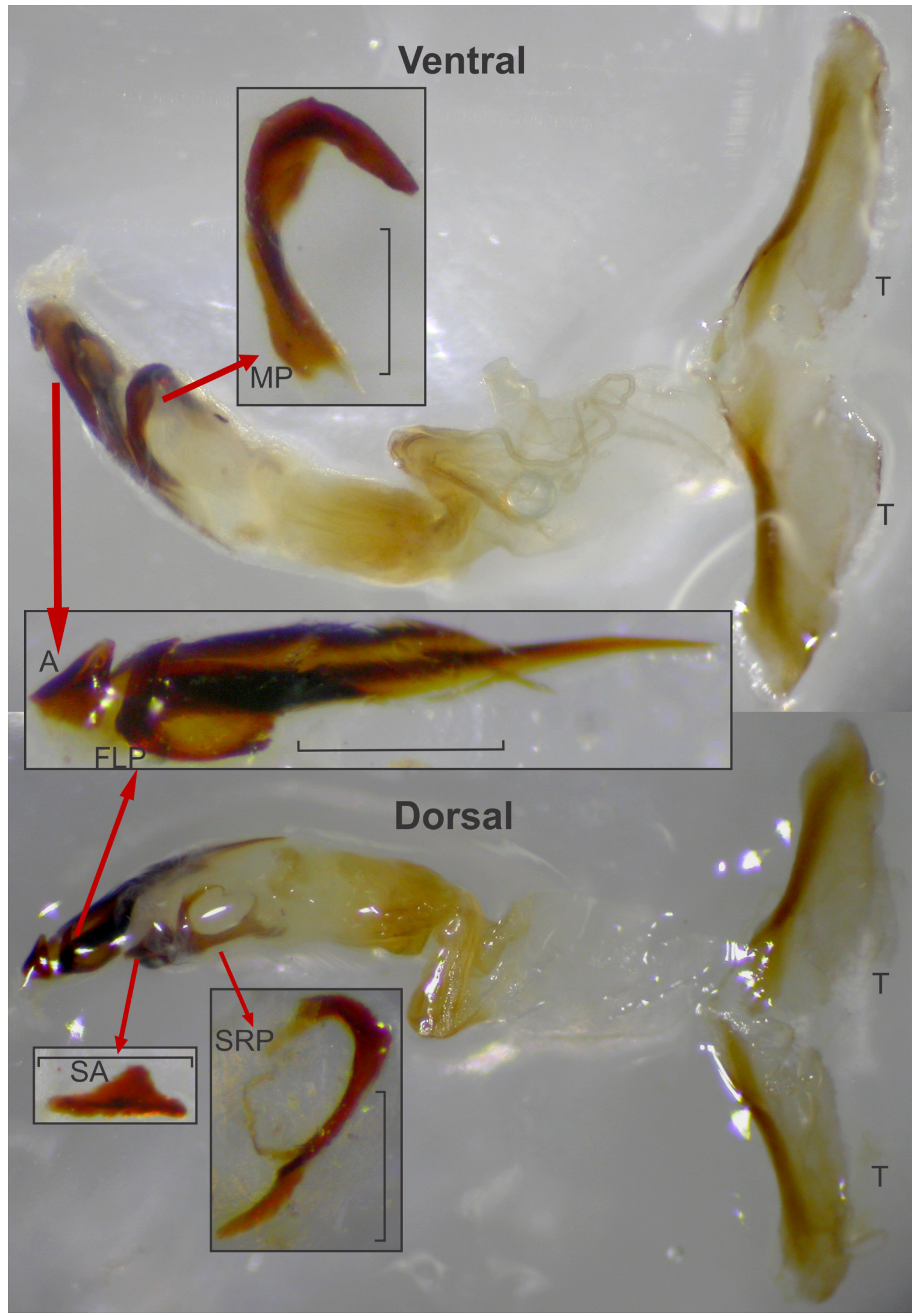

Fig. 6. Canthon (G.) smaragdulus smaragdulus (Fabricius, 1781), position of the sclerites of the internal sac. Abbreviations: see Material and methods. Scale bars: $0.5 \mathrm{~mm}$. 

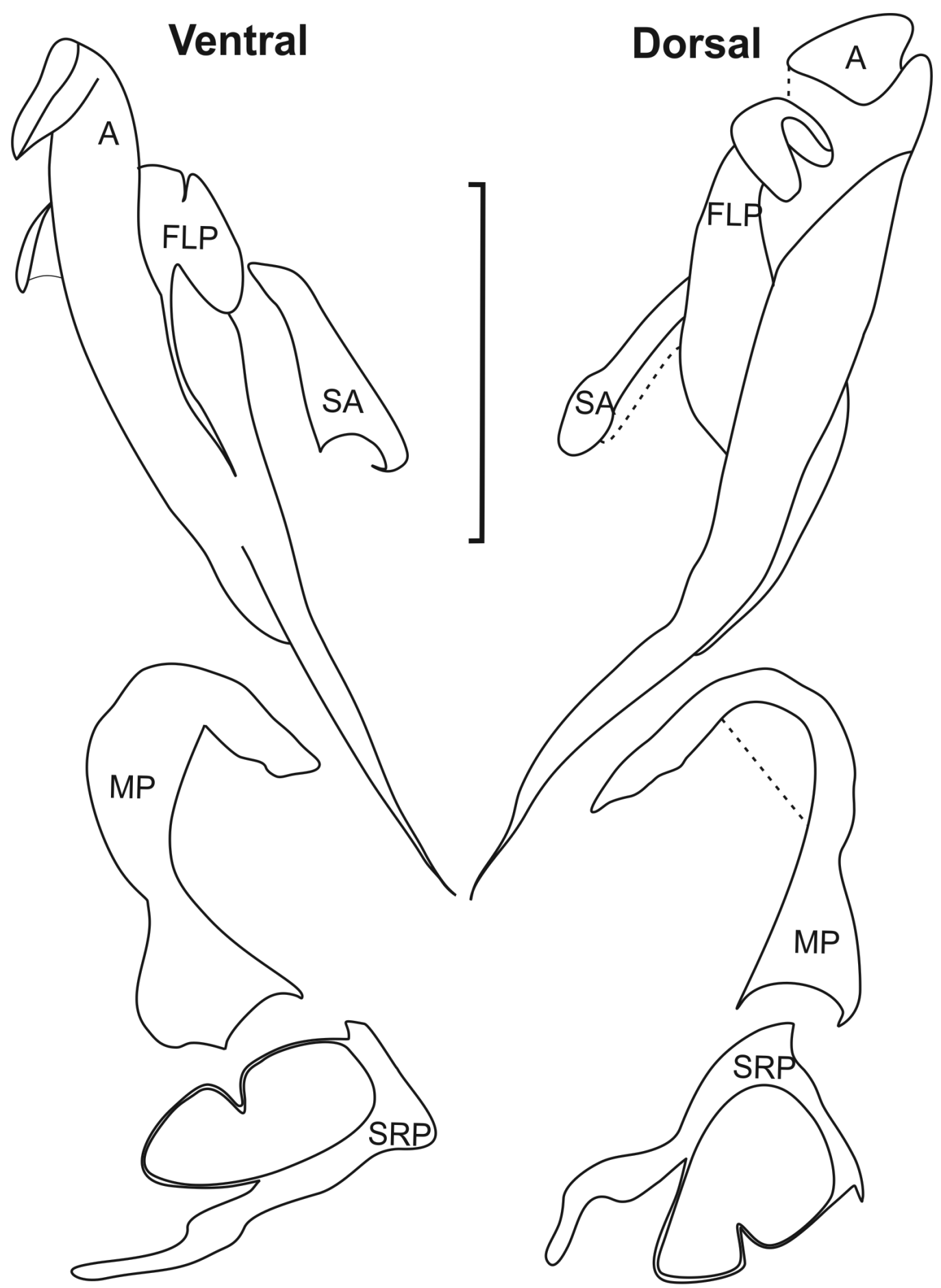

Fig. 7. Sclerites of the internal sac of Canthon (G.) fulgidus martinezi subsp. nov. Abbreviations: see Material and methods. Scale bar: $1.0 \mathrm{~mm}$. 
INTERNAL SAC (Fig. 9). FLP not completely involving A forming two duct with spiniform apex. MP distal to A, divided in MP 1 and MP 2, MP 1 semicircular, MP 1 fishhook-shaped with two hook; FLP + A complex involved by MP 2. SRP with lateral prolongation.

\section{Morphological variation}

BL: 10-13 mm, PW: 7-8.5 mm. Female protibial spur as wide as long, with apical U-shaped emargination (Fig. 4E). Pygidium convexity less protuberant in females than in males.

\section{Distribution}

North of the Amazon forest, in Venezuela, Guyana, Surinam, French Guiana and Brazil (state of Amapá) (Fig. 8).

\section{Taxonomic remarks}

This is the first time in the taxonomic history of Canthon that C. bicolor is considered part of the subgenus Goniocanthon. The following character combination supports this decision: metafemora not claviform, hypomeron lacking transverse keel and pygidium strongly convex, an exclusive character of Goniocanthon among the genus. The latter character was already cited by some authors for C. bicolor (e.g., Harold 1868; Schmidt 1922; Balthasar 1939). In a preliminary phylogenetic analysis, Medina et al. (2003) place this species close to the subgenera Goniocanthon and Peltecanthon, mainly due to male internal sac sclerites. The main morphological difference of C. bicolor among Goniocanthon

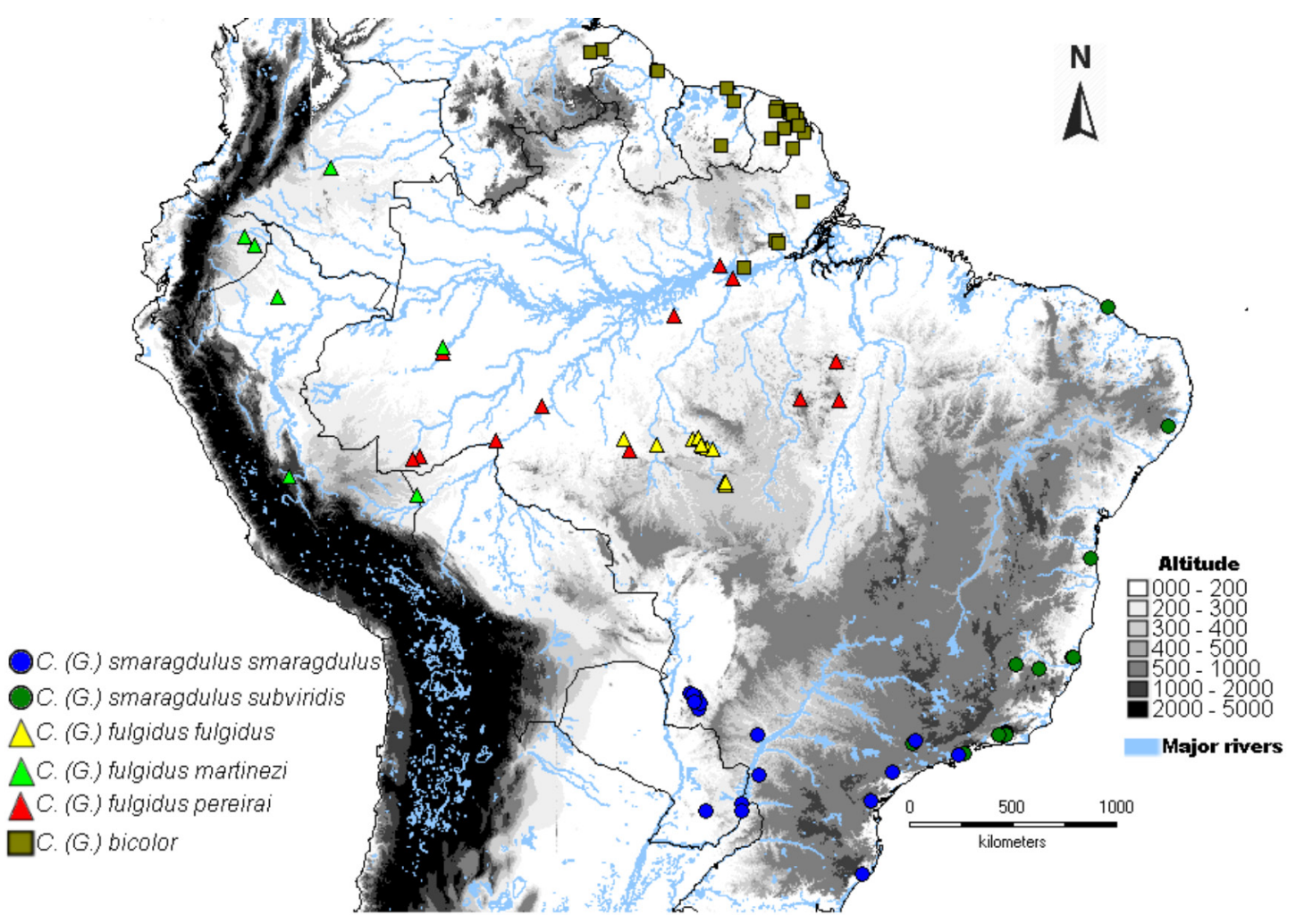

Fig. 8. Distribution map for subspecies of Goniocanthon: $C$. (G.) fulgidus martinezi subsp. nov. (green triangle); $C$. (G.) fulgidus pereirai subsp. nov. (red triangle); $C$. (G.) fulgidus fulgidus Redtenbacher, 1868 (yellow triangle); C. (G.) smaragdulus subviridis Schmidt, 1922 stat. rev. (green circle); C. (G.) smaragdulus smaragdulus (Fabricius, 1781) (blue circle) and C. bicolor Castelnau, 1840 (brown square). 

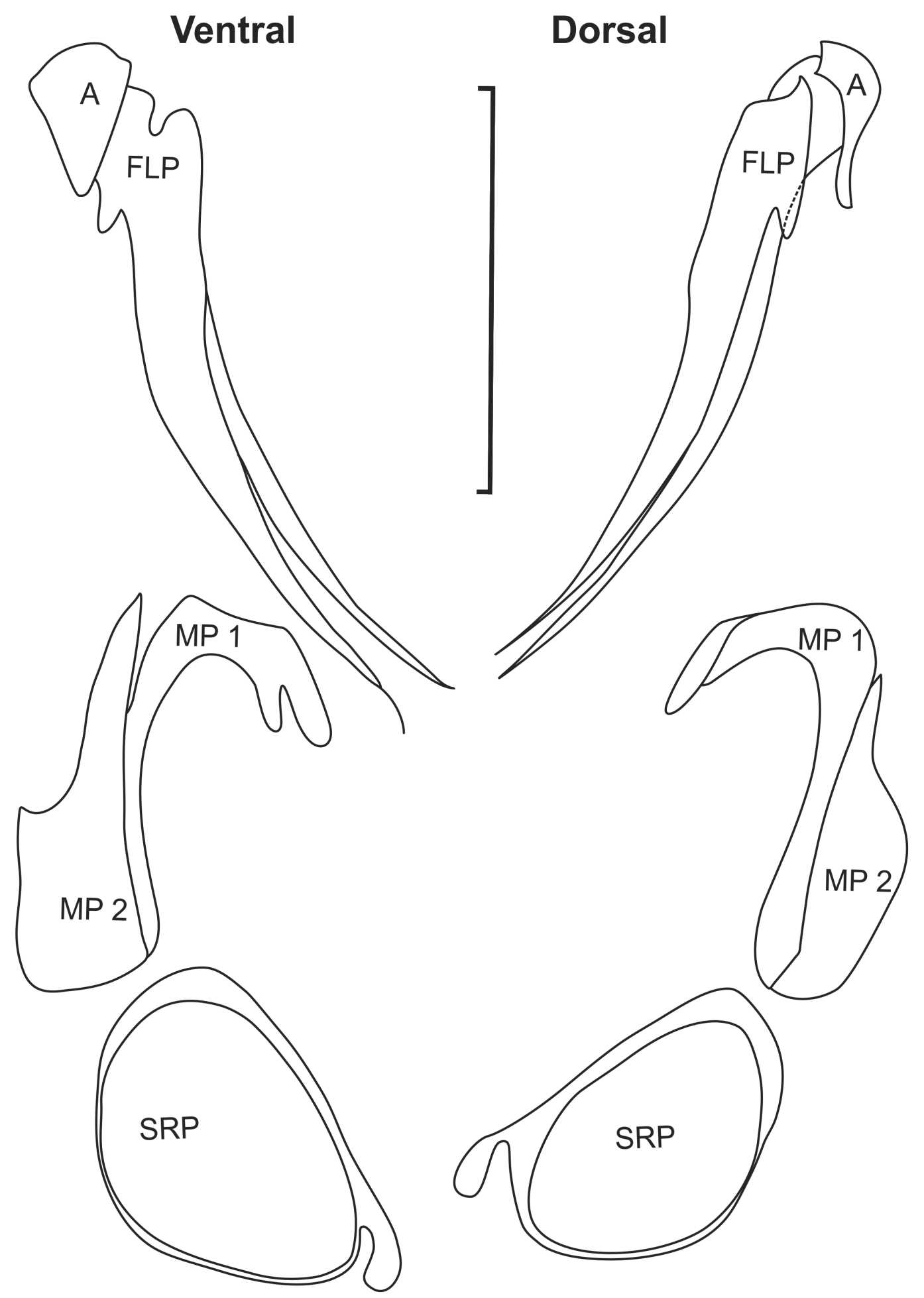

Fig. 9. Sclerites of the internal sac of Canthon (G.) bicolor Castelnau, 1840. Abbreviations: see Material and methods. Scale bar: $1.0 \mathrm{~mm}$. 
is the flat metasternum anterior lobe and the MP sclerite in the internal sac, which is divided in two structures (MP1 and MP2, Fig. 9). The relationship with Peltecanthon should be investigated with a more comprehensive phylogenetic analysis.

\section{Discussion}

Extensive taxonomic work dealing with Canthon and allied genera has been published in the last 50 years or so, when taxonomic knowledge of the Canthonini tribe (Deltochilini sensu Bouchard et al. 2011) was summarized. The subgenera of Canthon were the last defined (Halffter \& Martínez 1967, 1977). The division of species of Canthon is arranged into subgenera and incertae sedis species groups. Larger subgenera, such as Canthon s. str., Glaphyrocanthon and incertae sedis species groups, are difficult to diagnose and certainly need urgent taxonomic revisions. On the other hand, smaller subgenera such as Boreocanthon, Francmonrosia, Peltecanthon, Pseudepilissus and Goniocanthon have better morphological definitions. Even so, some of them still have taxonomic problems to be solved.

A convex metasternal anterior lobe and, most importanty, a strongly convex pygidium, are exclusive to Goniocanthon in the genus Canthon. Therefore, it should not be difficult to separate Goniocanthon from other subgenera of Canthon. The head margin, clypeal teeth, pronotal disc and dorso-ventral colour pattern of Goniocanthon resemble those of Peltecanthon and of some species of Canthon s. str. such as $C$. angularis Harold, 1868. However, none of them have a convex pygidium or metasternal anterior lobe. If Goniocanthon and Peltecanthon were sympatric, they would have similar chromatic variation

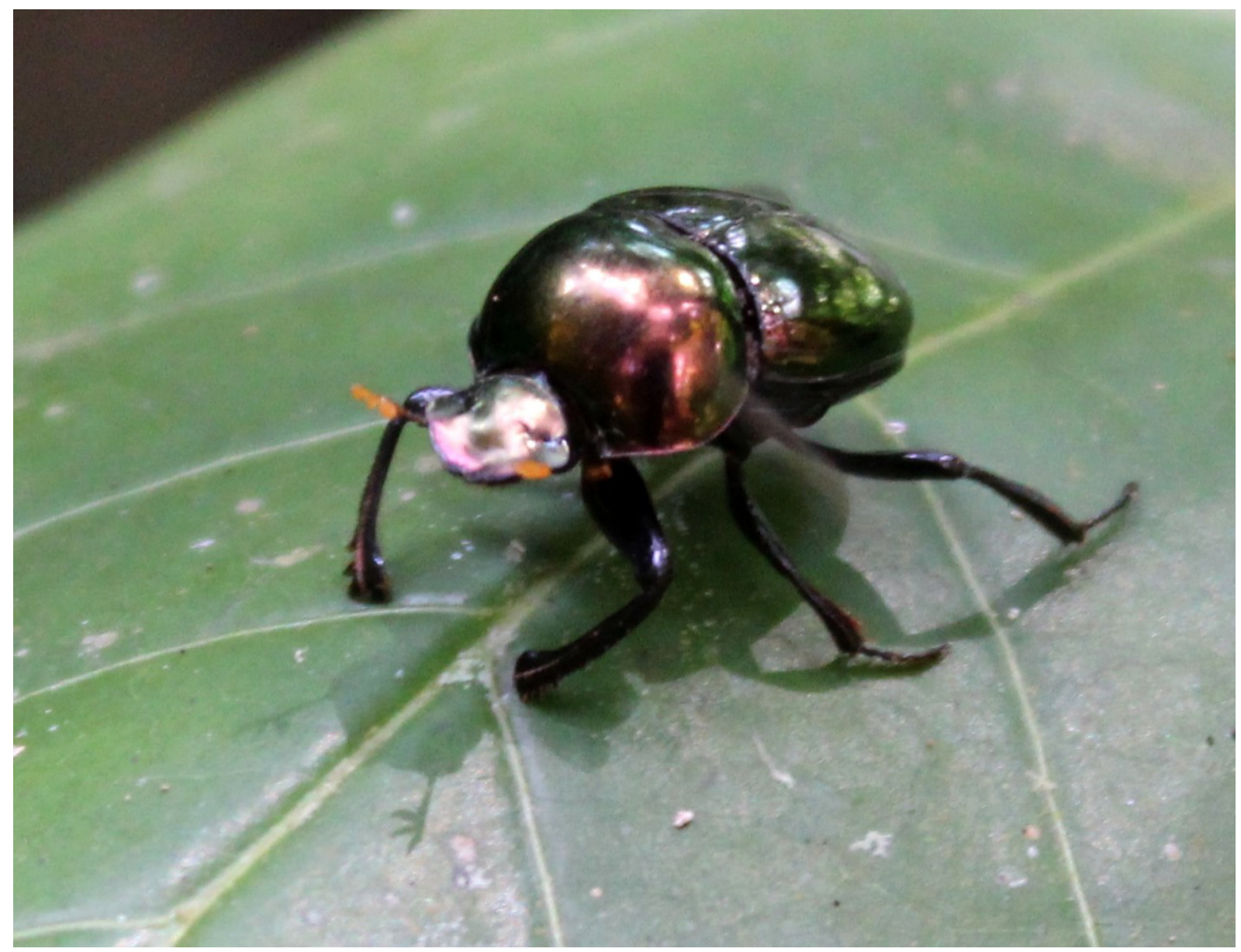

Fig. 10. Canthon (G.) fulgidus fulgidus Redtenbacher, 1868 showing perching behaviour. 
patterns, as is the case with other genera such as Dichotomius and Canthidium (authors' observations). The tooth on the anterior border of the profemur is shared with Francmonrosia (Medina et al. 2003), although it is absent in C. bicolor. Since the genus Canthon is evidently polyphyletic (Medina et al. 2003), we still cannot draw major conclusions on the phylogenetic relationships of Goniocanthon and other groups of Canthon.

The dorsal surface colouration of Goniocanthon may be problematic for the identification of subspecies, especially when separating Canthon fulgidus martinezi subsp. nov. and C. smaragdulus subviridis stat. rev. (Pessôa \& Lâne 1941; Blackwelder 1944; Vulcano \& Pereira 1964; Medina et al. 2001; Ratcliffe et al. 2015). In this case, one should examine the ventral surface of the meso- and metafemora (rugose in subspecies of $C$. fulgidus, smooth in C. smaragdulus) and the convexity of the metasternal anterior lobe (stronger in subspecies of C. smaragdulus than in C. fulgidus). Besides, C. fulgidus is Amazonian while $C$. smaragdulus occurs in the Atlantic Forest. For practical subspecies differentiation, one should associate dorsal colouration with geographic distribution. Even the internal sac sclerites do not provide this separation in subspecies, only showing interspecific variation between C. fulgidus, C. smaragdulus and C. bicolor.

The distribution of subspecies of $C$. smaragdulus follows a similar pattern of that of other Atlantic Forest taxa, which have distributions influenced by the Doce River (Cabanne et al. 2007; Carnaval \& Moritz 2008; Colombi et al. 2010). Canthon smaragdulus smaragdulus tends to occur south of the Doce River, while C. smaragdulus subviridis stat. rev. tends to occur in the northern portion of the Atlantic Forest (Morrone 2014). However, this separation is not absolute since both subspecies occur in close proximity in the states of Rio de Janeiro and São Paulo.

The distribution of subspecies of Canthon fulgidus seems to be influenced by the interfluves of major Amazon rivers (Fig. 8). The distribution of $C$. fulgidus martinezi subsp. nov. is similar to that of other taxa from the Imerí, Napo, Ucayali and Rondônia biogeographic provinces described by Morrone (2014). Canthon fulgidus pereirai subsp. nov. occurs in the states of Pará and Amazonas, distributed throughout the northern region of Madeira and Xingu-Tapajós Provinces. The distribution of C. fulgidus fulgidus covers the northern part of the state of Mato Grosso, corresponding to the southern part of the Madeira and Xingu-Tapajós Provinces according to Morrone (2014). Canthon bicolor occurs in the Guyana Shield, distributed throughout the Guianan Lowlands (Morrone 2014).

The perching behavior of C. fulgidus fulgidus (Fig. 10) shows how much this species is adapted to várzea forest habitats, which are annually flooded (Nunes-da-Cunha et al. 2015). Our observations corroborate two hypotheses of Louzada (1998) for perching in Scarabaeinae. The first one is related to perching for body temperature regulation, where $C$. fulgidus has been observed basking in the sun on larger leaves. The second hypothesis is related to resource foraging: after perching, many individuals located some kind of faecal mass, or followed us in the field while we installed baited traps with human faeces.

\section{Conclusions}

This work dealt with the taxonomic revision of Canthon (Goniocanthon) Pereira \& Martínez, 1956. We introduced C. bicolor and clarified the differentiation between C. fulgidus and C. smaragdulus, defining three and two subspecies for them, respectively. In this work, we proposed the hypothesis that body colour is influenced by geographic distribution. However, we strongly believe that our hypothesis needs the following tests: 1) collection of more geographical records and consequently morphological data to determine if species separation follows distribution and 2) comparison of morphological and molecular data to determine if the latter corroborates the morphological-geographical separation. Both approaches may lead to interesting future studies. 


\section{Acknowledgments}

LGOAN (23108.096582/2015-11) and RVN (23108.036500/13-3) received CAPES grants. FZVM received financial support from $\mathrm{CNPq}$ (process $\mathrm{n}^{\circ}$ 302997/2013-0, 484035/2013-4, 440604/20150, 568005/2014 PRONEM, 405697/2013-9, 248299/2012-3), Synthesis (GB-TAF-3855), INCTCENBAM/CNPq, PPBio, INCT-CENBAM and Rede ComCerrado/MMA. We thank all curators named in the methodology section, and our friend, the eminent taxonomist Mario Cupello, for selective revision and suggestions towards this manuscript, and for providing geographical records of C. bicolor.

\section{References}

Balthasar V. 1939. Eine Vorstudie zur Monographie der Gattung Canthon Hffsg. Folia Zoologica et Hydrobiologica 9 (2): 179-238.

Balthasar V. 1941. Scarabaeidae Laparostictae (Col.). Beitrag Fauna Perus 1: 337-358.

Balthasar V. 1951. Scarabaeidae Laparostictae (Col.). Beitrag Fauna Perus 2: 322-344.

Blackwelder R. 1944. Checklist of the coleopterous insects of Mexico, Central America the West Indies and South America. Part 2. Bulletin of the United States National Museum 185: 189-341.

Blanchard E. 1846. Insectes de l'Amérique méridionale. In: Blanchard E. \& Brullé G.A. (eds) Voyage dans L'Amérique Méridionale (le Brésil, la République orientale de L'Uruguay, la République Argentine, la Patagone, la République du Chili, la République de Bolivia, la République du Pérou), exécuté pendant les années 1826, 1827, 1828, 1829, 1830, 1831, 1832 et 1833, par Alcide D'Orbigny. Tome Sixième. 2. ${ }^{\text {}}$ partie: Insectes: 105-222. P. Bertrand, Paris \& Ve. Lerrault, Strasbourg.

https://doi.org/10.5962/bhl.title.85973

Bouchard P., Bousquet Y., Davies A.E., Alonso-Zarazaga M.E., Lawrence J.F., Lyal C.H.C., Newton A.F., Reid C.A.M., Schmitt M., Ślipiński S.A. \& Smith A.B.T. 2011. Family-group names in Coleoptera (Insecta). Zookeys 88 : 1-972. https://doi.org/10.3897/zookeys.88.807

Boucomont A. 1928. Wissenschaftliche Ergebnisse der schwedischen entomologischen Reise des Herrn Dr. A. Roman in Amazonas 1914-1915 (und 1923-1924), 12. Scarabaeidae. Arkiv fur Zoologi 19A (22): $1-8$.

Brullé G.A. 1838. Histoire naturelle des insectes, comprenant leur classification, leurs moeurs et la description des espèces. In: Audouin M.V. \& Brullé A. (eds) Histoire naturelle des insectes, comprenant leur classification, leurs moeurs, et la description des espèces. Tome VI. Coléoptères III. F.D. Pillot, Paris.

Cabanne G.S., Santos F.R. \& Miyaki C.Y. 2007. Phylogeography of Xiphorhynchus fuscus (Passeriformes, Dendrocolaptidae): vicariance and recent demographic expansion in southern Atlantic forest. Biological Journal of the Linnean Society 91: 73-84. https://doi.org/10.1111/j.1095-8312.2007.00775.x

Carnaval A.C. \& Moritz C. 2008. Historical climate modeling predicts patterns of current biodiversity in the Brazilian Atlantic forest. Journal of Biogeography 35: 1187-1201.

Castelnau Comte de. 1840. Histoire naturelle des insectes coléoptères. Tome Deuxième. Société bibliophile, Paris. https://doi.org/10.5962/bhl.title.36538

Colombi V.H., Lopes S.R. \& Fagundes V. 2010. Testing the Rio Doce as a riverine barrier in shaping the Atlantic rainforest population divergence in the rodent Akodon cursor. Genetics and Molecular Biology 33 (4): 785-789. https://doi.org/10.1590/S1415-47572010000400029

Evenhuis N. 2012. François-Louis Comte de Castelnau (1802-1880) and the mysterious disappearance of his original insect collection. Zootaxa 3168: 53-63. 
NUNES L.G. DE O.A. et al., Revision of the South American subgenus Canthon (Goniocanthon)

Fabricius J.C. 1781. Species insectorum exhibentes eorum differentias specificas, synonyma auctorum, loca Natalia, metamorphosin adiectis observationibus, descriptionibus. Tom I. Carol Ernest, Hamburg and Kiel. https://doi.org/10.5962/bhl.title.36509

Fabricius J.C. 1787. Mantissa Insectorum, sistens corum species nuper detectas. adjectis characteribus genericis, differentiis specificis, emendationibus, observationibus. Tom. I. Hafniae, Paris.

Fabricius J.C. 1792. Entomologia systematica emendata et aucta. Secundum classes, ordines, genera, species adjectis synonimis, locis, observationibus, descriptionibus. Tom I. Hafniae, Paris. https://doi.org/10.5962/bhl.title.122153

Fabricius J.C. 1801. Systema eleutheratorum secundum ordines, genera, species adiectis synonyms, locis, observationibus, descriptionibus. Tomus I. Kiliae Imprensis Bibliopolli Academici Novi, Paris.

Gacharná G.C. 1951. Catalogueo de los Coleopteros Colombianos. Revista de la Academia Colombiana de Ciencias Exactas, Físicas y Naturales 8: 221-229.

Gemminger M. \& Harold E. 1869. Catalogus coleopterorum hucusque descriptorum synonymicus et systematicus. Tome IV: Scarabaeidae. Sumptu E.H. Gummi, Monaco.

https://doi.org/10.5962/bhl.title.9089

Gillet J.J.E. 1911. Coleopterorum catalogus. Pars 38: Scarabaeidae: Coprinae. I. W. Junk, Berlin.

Guérin J. 1953. Coleopteros do Brasil. Faculdade de Filosofia, Ciências e Letras de São Paulo, São Paulo.

Halffter G. 1961. Monografia de las especies norteamericanas del genero Canthon. Ciencia, Revista Hispano-Americana de Ciencias Puras y Aplicadas 20 (9-12): 225-320.

Halffter G. \& Martínez A. 1967. Revisión monográfica de los Canthonina americanos (Coleoptera, Scarabaeidae) (2 $2^{\mathrm{a}}$ Parte). Revista de la Sociedad Mexicana de Historia Natural 28 (2): 79-117.

Halffter G. \& Martínez A. 1977. Revisión monográfica de los Canthonina americanos, IV parte. Clave para géneros y subgéneros. Folia Entomológica Mexicana. 38: 29-107.

Harold E. 1868. Monographie der Gattung Canthon. Berliner entomologische Zeitschrift 12: 1-144.

Harold E. 1875. Verzeichniss der von Dr. Teuscher in Cantagallo gesammelten coprophagen Lamellicornien. Coleopterologische Hefte 13: 57-72.

Heyne A. \& Taschenberg O. 1908. Die exotischen Käfer in Wort und Bild. J.F. Schreiber, Esslingen/Munich. https://doi.org/10.5962/bhl.title.9363

ICZN (International Commission on Zoological Nomenclature) 1999. International Code of Zoological Nomenclature. Fourth Edition adopted by the International Union of Biological Sciences. International Trust for Zoological Nomenclature, London.

Jablonsky C.G. \& Herbst J.F.W. 1789. Natursystem aller bekannten in- und ausländischen Insekten, als eine Fortsetzung der von Büffon'schen Naturgeschichte. Nach dem System des Ritters von Linné und Fabricius zu bearbeiten angefangen. Der Käfer zweiter Theil. Mit siebzehn illuminirten Kupfertafeln. Heft 2. Joachim Pauli, Berlin.

Lange R.B. 1947. Ensaio da zoogeografia dos scarabaeidae do Paraná com algumas notas eto-ecológicas. Arquivos Museu Paranaense 6: 305-315.

Louzada J.N.C. 1998. Considerations on the perching behaviour of tropical dung beetles (Coleoptera, Scarabaeidae). Revista Brasileira de Entomologia 41 (2-4): 125-128.

Martínez A. 1959. Catalogueo de los Scarabaeidae Argentinos. Revista del Museo Argentino de Ciencias Naturales "Bernardino Rivadavia", Ciencias Zoológicas 5 (1): 1-126. 
Medina C.A., Lopera-Toro A., Vítolo A. \& Gill B. 2001. Escarabajos coprófagos (Coleoptera: Scarabaeidae: Scarabaeinae) de Colombia. Biota Colombiana 2 (2): 131-144.

Medina C.A., Scholtz C.H. \& Gill B.D. 2003. Morphological variation and systematics of Canthon Hoffmansegg 1817, and related genera of New World Canthonini dung beetles (Coleoptera, Scarabaeinae). Deutsche entomologische Zeitschrift 50 (1) : 23-68.

Morrone J.J. 2014. Biogeographical regionalization of the Neotropical region. Zootaxa 3782 (1): 1-110. https://doi.org/10.11646/zootaxa.3782.1.1

Nunes-da-Cunha C., Piedade M.T.F. \& Junk W.J. (eds). 2015. Classificação e delineamento das áreas úmidas brasileiras e de seus macrohabitats. EdUFMT, Cuiabá.

Olivier G.A. 1789. Entomologie, ou histoire naturelle des insects, avex leurs caractères génériques et spécifiques, leur description, leur synonymie, et leur figure enluminée. Coléoptères. Tome premier. Baudouin, Paris. https://doi.org/10.5962/bhl.title.49479

Papavero N. (eds). 1971. Essays on the History of Neotropical Dipterology, with Special Reference to Collectors (1750-1905). Vol 1. EGRT Press, São Paulo. https://doi.org/10.5962/bhl.title.101715

Pereira F.S. \& Martínez A. 1956. Os gêneros de Canthonini americanos (Col. Scarabaeidae). Revista Brasileira de Entomologia 6: 91-192.

Pessôa S.B. \& Lâne F. 1941. Coleopteros necrófagos de interesse médico-legal. Ensáio monográfico sobre a família Scarabaeidae de São Paulo e regiões vizinhas. Arquivos de Zoologia do Estado de São Paulo 2: 389-504.

Ratcliffe B.C., Jameson M.L., Figueroa L., Cave R.D., Paulsen M.J., Cano E.B., Beza-Beza C., Jimenez-Ferbans L. \& Reyes-Castillo P. 2015. Beetles (Coloeptera) of Peru: a survey of the families. Scarabaeoidea. Journal of the Kansas Entomological Society 88 (2): 186-207. https://doi.org/10.2317/kent-88-02-186-207.1

Redtenbacher L. 1868. Reise der Österreichischen Fregatte Novara um die Erde in den Jahren 1857 , 1858, 1859 unter den Befehlen des Commodore B. von Wüllerstorf-Urbair. Zoologischer Theil. Zweiter Band. Coleopteren. Mit 5 Tafeln. Karl Gerold's Sohn, Vienna.

Schmidt A. 1920. Beitrag zur Kenntnis der Gattungen Canthon Hffsg., Sybax Boh., Aphodius Ill., Simogenius Har., Ataenius Har. Archiv für Naturgeschichte A 86 (9): 114-147.

Schmidt A. 1922. 1. Bestimmungstabelle der mir bekannten Canthon-Arten. 2. Verbreitungsgebiete der Canthon-Arten. 3. Neubeschreibungen von Canthon, Saprosites, Mendidius, Euparia und Ataenius. Archiv für Naturgeschichte 88 (3): 61-103.

Scholtz C.H. 2009. Section C. Phylogeny of the Scarabaeinae. In: Scholtz C.H., Davis A.L.V. \& Kryger U. (eds) Evolutionary Biology and Conservation of Dung Beetles: 225-327. Pensoft Publishers, Sófia.

Staig R.A. 1931. The Fabrician Types of Insects in the Hunterian Collection at Glasgow University, Coleoptera, Part I. Cambridge University Press, Cambridge.

Tarasov S.I. \& Génier F. 2015. Innovative Bayesian and parsimony phylogeny of dung beetles (Coleoptera, Scarabaeidae, Scarabaeinae) enhanced by ontology-based partitioning of morphological characters. PLoS ONE 10 (3): 1-86. https://doi.org/10.1371/journal.pone.0116671

Tarasov S.I. \& Dimitrov D. 2016. Multigene phylogenetic analysis redefines dung beetles relationships and classification (Coleoptera: Scarabaeidae: Scarabaeinae). BioMed Central Evolutionary Biology 16: 257. https://doi.org/10.1186/s12862-016-0822-x 
Tarasov S.I. \& Solodovnikov A.Y. 2011. Phylogenetic analyses reveal reliable morphological markers to classify mega-diversity in Onthophagini dung beetles (Coleoptera: Scarabaeidae: Scarabaeinae). Cladistics 27: 1-39. https://doi.org/10.1111/j.1096-0031.2011.00351.x

Vaz-de-Mello F.Z. 2000. Estado atual de conhecimentos dos Scarabaeidae s. str. (Coleoptera: Scarabaeoidea) do Brasil. In: Martín-Piera F., Morrone J.J. \& Melic A. (eds) Hacya un proyecto CYTED para el inventario y estimación de la diversidad entomológica en Iberoamérica: PRIBES-2000: Vol.1: 183-195. Monografías Tercer Milenio y Sociedad Entomológica Aragonesa, Zaragoza.

Vaz-de-Mello F.Z., Edmonds W.D., Ocampo F. \& Schoolmeesters P. 2011. A multilingual key to the genera and subgenera of the subfamily Scarabaeinae of the New World (Coleoptera: Scarabaeidae). Zootaxa 2854: 1-73.

Vulcano M.A. \& Pereira F.S. 1964. Catalogue of the Canthonini (Col. Scarab.) inhabiting the Western Hemisphere. Entomologische Arbeiten aus dem Museum G. Frey 15 (2): 570-685.

Zunino M. 1978. L'armatura genitale negli Onthophagini: tecniche di preparazione e criteri di studio. L'informatore del giovane entomologo. Bollettino della Società Entomologica Italiana, Suppl. 90: 2126.

Manuscript received: 30 March 2017

Manuscript accepted: 5 December 2017

Published on: 23 May 2018

Topic editor: Gavin Broad

Section editor: Max Barclay

Desk editor: Kristiaan Hoedemakers

Printed versions of all papers are also deposited in the libraries of the institutes that are members of the EJT consortium: Muséum national d'Histoire naturelle, Paris, France; Botanic Garden Meise, Belgium; Royal Museum for Central Africa, Tervuren, Belgium; Natural History Museum, London, United Kingdom; Royal Belgian Institute of Natural Sciences, Brussels, Belgium; Natural History Museum of Denmark, Copenhagen, Denmark; Naturalis Biodiversity Center, Leiden, the Netherlands; Museo Nacional de Ciencias Naturales-CSIC, Madrid, Spain; Real Jardín Botánico de Madrid CSIC, Spain. 